P7-2003-32

В. И. Загребаев, В. В. Самарин

ОКОЛОБАРЬЕРНОЕ СЛИЯНИЕ ТЯЖЕЛЫХ ЯДЕР. СВЯЗЬ КАНАЛОВ

Направлено в журнал «Ядерная физика» 
Загребаев В. И., Самарин В. В.

P7-2003-32

Околобарьерное слияние тяжелых ядер. Связь каналов

В работе исследуется проблема квантового описания околобарьерного слияния тяжелых ядер, происходящего в условиях сильной связи относительного движения с вращением деформированных ядер и с динамической деформацией их поверхностей. Предложен новый эффективный метод решения системы связанных уравнений Шредингера с граничными условиями, соответствующими полному поглощению потока, прошедшего многомерный кулоновский барьер. Новый метод не содержит ограничений на число учитываемых каналов и позволяет рассчитывать сечения слияния очень тяжелых ядер, используемых для синтеза сверхтяжелых элементов. Совместный анализ рельефа многомерной потенциальной поверхности и многоканальной волновой функции вблизи кулоновского барьера дает наглядную интерпретацию динамике околобарьерного слияния ядер. Проведено сравнение с экспериментальными данными и с полуэмпирической моделью учета связи каналов. Расчетные программы размещены на web-сервере http://nrv.jinr.ru/nrv/ со свободным доступом.

Работа выполнена в Лаборатории ядерных реакций им. Г. Н. Флерова ОИЯИ.

Препринт Объединенного института ядерных исследований. Дубна, 2003

\section{Перевод авторов}

Zagrebaev V. I., Samarin V. V.

P7-2003-32

Near-Barrier Fusion of Heavy Nuclei. Coupling of the Channels

The problem of quantum description of near-barrier fusion of heavy nuclei taking place under strong coupling of relative motion with rotation of deformed nuclei and with dynamic deformations of their surfaces is studied in the paper. A new effective method is proposed for numerical solution of a set of coupled Schrödinger equations with boundary conditions corresponding to a full absorption of the flux penetrated through the multi-dimensional Coulomb barrier. The method has no limitation on the number of coupled channels and allows one to calculate fusion cross-sections of very heavy nuclei used for synthesis of super-heavy elements. A combined analysis of the multi-dimensional potential energy surface relief and the multi-channel wave function in the vicinity of the Coulomb barrier gives a clear interpretation of near-barrier fusion dynamics. Comparison with experimental data and with semi-empirical model calculations is performed. The computing codes are allocated at the web-server http://nrv.jinr.ru/nrv/ with free access.

The investigation has been performed at the Flerov Laboratory of Nuclear Reactions, JINR. 


\section{Введение}

Процессы околобарьерного слияния атомных ядер по-прежнему привлекают повышенное внимание теоретиков и экспериментаторов. Динамику низкоэнергетического процесса слияния определяет квантовое туннелирование через кулоновский барьер, происходящее в условиях сильной связи относительного движения с внутренними степенями свободы, главным образом, с колебаниями ядерных поверхностей, вращением деформированных ядер и передачей нуклонов [1]. Заметим, что эта теоретическая проблема является достаточно общей для многих областей физики и химии. Достигнутое в последнее время значительное улучшение экспериментальной техники в этой области привело к возможности прецизионных измерений, позволяющих экспериментально изучать детали и тонкие эффекты процесса подбарьерного слияния (см., например, [2,3], а также обзорную работу [4]). Точное решение соответствующей квантовой задачи (и/или ее квазиклассического аналога) сопряжено с определенными трудностями. В результате, несмотря на достаточно хорошее общее понимание физики всего процесса, в некоторых случаях мы попрежнему не имеем однозначной интерпретации экспериментальных данных. Еще хуже дело обстоит с предсказаниями сечений подбарьерного слияния для еще не изученных комбинаций тяжелых ядер, что особенно актуально для планирования и проведения дорогостоящих экспериментов по синтезу сверхтяжелых элементов.

В последние годы было предложено несколько алгоритмов численного решения системы связанных уравнений Шредингера, моделирующих связь каналов при околобарьерном слиянии тяжелых ядер и использующих либо приближенный метод диагонализации матрицы связи на барьере [5], либо непосредственное численное решение дифференциальных уравнений [6]. Как было показано в [7], учет реалистических ядро-ядерных сил приводит к достаточно большим динамическим деформациям тяжелых сталкивающихся ядер, для правильного описания которых требуется учитывать большое число возбуждаемых фононов. Следуя в основном работе [6], мы разработали новый алгоритм решения системы дифференциальных уравнений второго порядка, позволяющий избежать ограничения на число учитываемых каналов. Вторым отличием нашего подхода является более аккуратное рассмотрение граничных условий на падающий поток, т.е. обеспечение полного отсутствия отраженных волн из области за барьером. Наряду с проницаемостью барьера, это позволяет вычислять также и саму многомерную волновую функцию в околобарьерной области, которую можно использовать для более глубокого понимания и детального анализа динамики многомерного туннелирования. Отказываясь, как и в [6], от линейного приближения по связывающему взаимодействию, мы, в отличие от [6], используем явный (квадратурный) метод расчета матричных элементов взаимодействия, обеспечивающий заданную точность, не зависящую от числа учитываемых каналов. Развитый подход использован для анализа процессов слияния статически деформированных и сферических деформируемых тяжелых ядер. Проведено сравнение с экспериментальными данными и с результатами полуэмпирической модели учета связи каналов в процессах слияния. 


\section{1. Взаимодействие деформированных ядер}

Форма деформированного аксиально-симметричного ядра определяется формулой

$$
R(\vec{\beta}, \theta)=\tilde{R} \cdot\left(1+\sum_{\lambda \geq 2} \beta_{\lambda} \sqrt{\frac{2 \lambda+1}{4 \pi}} P_{\lambda}(\cos \theta)\right),
$$

где $\vec{\beta} \equiv\left\{\beta_{\lambda}\right\}$ - безразмерные параметры деформации мультипольности $\lambda=2,3, \ldots$, $P_{\lambda}$ - полиномы Лежандра,

$$
\tilde{R}=R_{0}\left[1+\frac{3}{4 \pi} \sum_{\lambda} \beta_{\lambda}^{2}+\frac{1}{4 \pi} \sum_{\lambda, \lambda^{\prime}, \lambda^{\prime \prime}} \sqrt{\frac{\left(2 \lambda^{\prime}+1\right)\left(2 \lambda^{\prime \prime}+1\right)}{4 \pi(2 \lambda+1)}}\left(\lambda^{\prime} 0 \lambda^{\prime \prime} 0 \mid \lambda 0\right)^{2} \beta_{\lambda} \beta_{\lambda^{\prime}} \beta_{\lambda^{\prime \prime}}\right]^{-1 / 3},
$$

$R_{0}$ - радиус эквивалентной сферы с тем же объемом, что и объем деформированного ядра, а $\left(\lambda^{\prime} 0 \lambda^{\prime \prime} 0 \mid \lambda 0\right)$ - коэффициенты Клебша-Гордона. Потенциальная энергия взаимодействия двух деформируемых ядер может быть записана в виде суммы кулоновской и ядерной энергии и энергии деформации в гармоническом приближении:

$$
V_{12}\left(r ; \vec{\beta}_{1}, \theta_{1}, \vec{\beta}_{2}, \theta_{2}\right)=V_{C}\left(r ; \vec{\beta}_{1}, \theta_{1}, \vec{\beta}_{2}, \theta_{2}\right)+V_{N}\left(r ; \vec{\beta}_{1}, \theta_{1}, \vec{\beta}_{2}, \theta_{2}\right)+\frac{1}{2} \sum_{i=1}^{2} \sum_{\lambda} C_{i \lambda} \cdot\left(\beta_{i \lambda}-\beta_{i \lambda}^{\text {g.s. }}\right)^{2} .
$$

Здесь и далее индекс $i=1,2$ нумерует взаимодействующие ядра, $C_{i \lambda}$ - параметры жесткости ядерной поверхности, а $\theta_{1,2}-$ ориентации осей симметрии деформированных ядер (рис. 1).

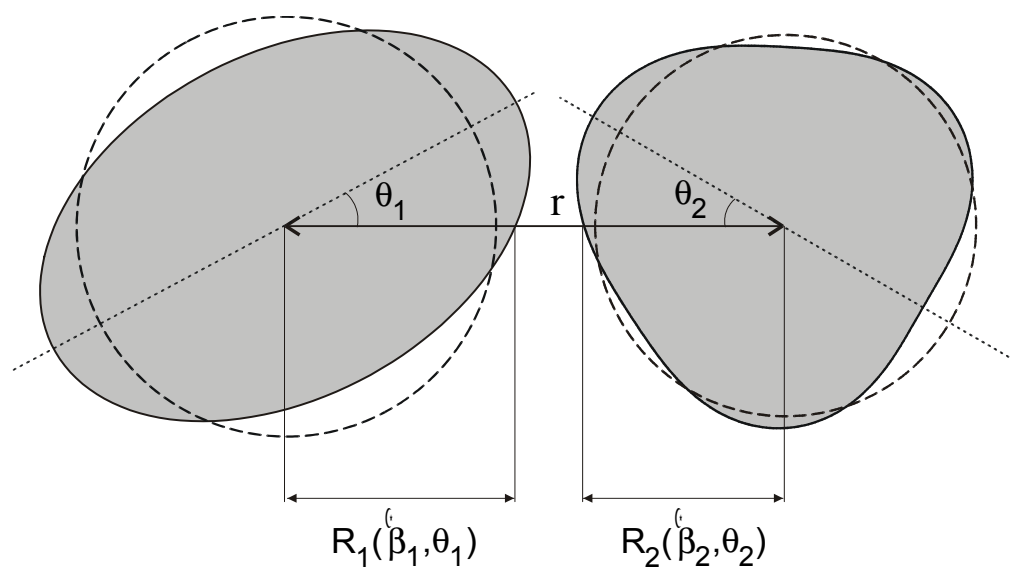

Рис. 1. Схематическое изображение относительного положения двух деформированных ядер, вращающихся в плоскости реакции

Пренебрегая мультиполь-мультипольным взаимодействием, с точностью до второго порядка по деформациям кулоновское взаимодействие деформированных ядер можно записать в виде 


$$
\begin{gathered}
V_{C}=Z_{1} Z_{2} e^{2}\left[F^{(0)}(r)+\sum_{i=1}^{2} \sum_{\lambda \geq 2} F_{i \lambda}^{(1)}(r) \beta_{i \lambda} Y_{\lambda 0}\left(\theta_{i}\right)\right]+ \\
+Z_{1} Z_{2} e^{2} \sum_{i=1}^{2} \sum_{\lambda^{\prime}} \sum_{\lambda^{\prime \prime}} \sum_{\lambda=\left|\lambda^{\prime}-\lambda^{\prime \prime}\right|}^{\lambda+\lambda^{\prime \prime}} F_{i \lambda}^{(2)}(r) \sum_{\mu} \int Y_{\lambda^{\prime} \mu}^{*} Y_{\lambda^{\prime \prime}-\mu}^{*} Y_{\lambda 0} d \Omega \cdot \beta_{i \lambda^{\prime}} \beta_{i \lambda^{\prime}} Y_{\lambda 0}\left(\theta_{i}\right)+\ldots
\end{gathered}
$$

Здесь $F_{\lambda}^{(n)}(r)$ - формфакторы взаимодействия. При $r>R_{1}+R_{2} \quad F^{(0)}=\frac{1}{r}$, $F_{i \lambda}^{(1)}=\frac{3}{2 \lambda+1} \frac{R_{i}^{\lambda}}{r^{\lambda+1}}, \quad F_{i \lambda=2}^{(2)}=\frac{6}{5} \frac{R_{i}^{2}}{r^{3}}, \quad F_{i \lambda=4}^{(2)}=\frac{R_{i}^{4}}{r^{5}}$. При меньших значениях $r$, когда ядерные поверхности перекрываются, для формфакторов $F_{\lambda}^{(n)}(r)$ получаются более сложные выражения [8], что, впрочем, несущественно для рассматриваемых здесь процессов слияния, поскольку положение кулоновского барьера $R_{C}^{B}>R_{1}+R_{2}$. При описании вращения деформированных ядер обычно учитывается их квадрупольная и/или гексадекапольная деформация. Поскольку, как правило, $\beta_{4} \ll 1$, то в третьем слагаемом сохраняются лишь члены с $\lambda^{\prime}=\lambda^{\prime \prime}=2$ и $\lambda$ принимает значения 2 и 4 .

Короткодействующее ядерное взаимодействие зависит от расстояния между поверхностями ядер, в качестве которого обычно используется расстояние вдоль межъядерной оси $\xi=r-R_{1}\left(\vec{\beta}_{1}, \theta_{1}\right)-R_{2}\left(\vec{\beta}_{2}, \theta_{2}\right)$ или минимальное расстояние между поверхностями (см. рис. 1). Это взаимодействие часто аппроксимируется потенциалом Вудса-Саксона, который записывается в форме $V_{W S}(\xi)=V_{0}[1+\exp (\zeta / a)]^{-1}, \quad$ где $\quad \zeta=r-R_{V}-\Delta R_{1}-\Delta R_{2}, \quad \Delta R_{1}=R_{1}\left(\vec{\beta}_{1}, \theta_{1}\right)-R_{1}$, $\Delta R_{2}=R_{2}\left(\vec{\beta}_{2}, \theta_{2}\right)-R_{2}$. Заметим, что для потенциала Вудса-Саксона радиус взаимодействия $R_{V}=r_{0}^{V}\left(A_{1}^{1 / 3}+A_{2}^{1 / 3}\right)$ обычно не совпадает с суммой радиусов самих ядер и $r_{0}^{V}$ является дополнительным независимым параметром. Другой возможностью является использование потенциала "proximity" для описания ядерного взаимодействия [9]

$$
V_{p r o x}(\xi)=4 \pi \gamma b P_{s p h}^{-1} \cdot \Phi(\xi / b)
$$

Здесь $\Phi(\xi / b)$ - универсальный безразмерный формфактор, $b$ - параметр толщины поверхностного слоя $(\approx 1$ ФМ $), \quad \gamma=\gamma_{0}\left(1-1.7826 \cdot I^{2}\right), \quad \gamma_{0}=0.95 \mathrm{MэB} \mathrm{Фм}^{-2}-$ коэффициент поверхностного натяжения, $I=(N-Z) / A$, $\xi=r-R_{1}\left(\vec{\beta}_{1}, \theta_{1}\right)-R_{2}\left(\vec{\beta}_{2}, \theta_{2}\right), \quad$ a $\quad P_{s p h}=1 / \bar{R}_{1}+1 / \bar{R}_{2} \quad$ и $\quad \bar{R}_{i}=R_{i}\left[1-\left(b / R_{i}\right)^{2}\right] . \quad$ Это взаимодействие наиболее чувствительно к выбору материальных радиусов ядер. Наиболее реалистические результаты получаются при выборе $r_{0} \approx 1.16$ Фм для радиусов тяжелых ядер $(A>40)$ и $r_{0} \approx 1.22$ Фм для ядер с $A \sim 16$. Основное достоинство потенциала "proximity" состоит в его универсальности, т.е. в отсутствии подгоночных параметров типа $V_{0}, r_{0}^{V}, a_{V}$. 
Величина притяжения двух ядерных поверхностей зависит также от их кривизны $[9,10]$, т.е. от площади соприкасающихся поверхностей. Обычно это учитывается заменой $P_{s p h}$ в (5) на выражение

$$
P\left(\vec{\beta}_{1}, \theta_{1}, \vec{\beta}_{2}, \theta_{2}\right)=\left[\left(k_{1}^{\|}+k_{2}^{\|}\right)\left(k_{1}^{\perp}+k_{2}^{\perp}\right)\right]^{1 / 2},
$$

где $k_{i}^{\|, \perp}$ - главные параметры локальной кривизны поверхностей снаряда и мишени (см., например, [11]). Для сферических ядер $k_{i}^{\|, \perp}=R_{i}^{-1}$ и $P=P_{s p h}$. В случае межосевых динамических деформаций $\left(\theta_{1}=\theta_{2}=0\right)$, возникающих при медленных столкновениях динамически деформируемых ядер, локальная кривизна поверхностей может быть найдена в явном виде (см. приложение 1)

$P\left(\vec{\beta}_{1}, \theta_{1}=0, \vec{\beta}_{2}, \theta_{2}=0\right)=\sum_{i=1,2} \frac{1}{\tilde{R}_{i}}\left(1+\sum_{\lambda \geq 2} \sqrt{\frac{2 \lambda+1}{4 \pi}} \beta_{i \lambda}\right)^{-2}\left(1+\sum_{\lambda \geq 2}(1+\eta(\lambda)) \sqrt{\frac{2 \lambda+1}{4 \pi}} \beta_{i \lambda}\right)$,

где $\eta(\lambda)=3 \cdot 4 \cdot \ldots \cdot(\lambda+1) /(\lambda-1)$ !. Для деформированных вращающихся ядер, в принципе, необходимо учитывать отличие кратчайшего расстояния между поверхностями $\xi_{S}$ от расстояния $\xi$, вычисленного вдоль центральной линии (см. рис. 1). Однако для реалистических деформаций результирующий эффект учета неравенства $\xi_{S}$ и $\xi$ при вычислении потенциалов взаимодействия и сечений слияния не очень велик по сравнению с эффектом изменения кривизны $\left(P \neq P_{s p h}\right)$ [12].

Формально выражение (6) может обращаться в ноль при некоторых отрицательных значениях деформации (соприкосновение двух плоских поверхностей). Этот нефизический эффект возникает из-за пренебрежения конечными размерами площадей соприкасающихся поверхностей ядер и указывает на необходимость перехода к более точному приближению при больших отрицательных деформациях. Основной вклад в ядро-ядерный потенциал вносят взаимодействия наиболее близко расположенных нуклонов, число которых хотя и зависит от локальной кривизны поверхностей, но всегда конечно. Таким образом, вместо простой замены в (5) $P_{s p h}$ на величину $P$ для короткодействующего межъядерного взаимодействия более правильно использовать выражение $V_{N}=G\left(\vec{\beta}_{1}, \theta_{1}, \vec{\beta}_{2}, \theta_{2}\right) \cdot V_{N}^{0}\left(r, \vec{\beta}_{1}, \theta_{1}, \vec{\beta}_{2}, \theta_{2}\right)$, где $V_{N}^{0}$ взаимодействие, вычисленное $\mathrm{c}$ учетом деформаций ядер и их взаимной ориентации, но без учета изменения кривизны поверхностей, а $G\left(\vec{\beta}_{1}, \theta_{1}, \vec{\beta}_{2}, \theta_{2}\right)$ геометрический фактор, учитывающий изменение числа взаимодействующих нуклонов, находящихся в близко расположенных поверхностных слоях двух ядер, по сравнению со случаем сферических поверхностей. В приложении 2 приводится вывод приближенного выражения для геометрического фактора $G\left(\vec{\beta}_{1}, \theta_{1}, \vec{\beta}_{2}, \theta_{2}\right)$, который играет заметную роль при не очень малых деформациях.

Жесткость деформации ядерной поверхности $C_{\lambda}$ может быть найдена из экспериментального значения вероятности электромагнитного перехода $B(E \lambda)$ из состояния с возбуждением одного вибрационного кванта [13]: 


$$
C_{\lambda}=(2 \lambda+1) \frac{\varepsilon_{\lambda}}{2<\beta_{\lambda}^{0}>^{2}}
$$

Здесь $\varepsilon_{\lambda}=\hbar \omega_{\lambda}$ - энергия колебаний, $<\beta_{\lambda}^{0}>=\frac{4 \pi}{3 Z R_{0}^{\lambda}}\left[\frac{B(E \lambda)}{e^{2}}\right]^{1 / 2}$ - среднеквадратичное значение полной деформации при нулевых колебаниях. B отсутствие соответствующих экспериментальных данных параметры колебаний ядерной поверхности могут быть определены в модели жидкой капли [13]:

$$
\begin{gathered}
C_{\lambda}^{L D}=\gamma_{0} R_{0}^{2}(\lambda-1)(\lambda+2)-\frac{3}{2 \pi} \frac{Z e^{2}}{R_{0}} \frac{(\lambda-1)}{(2 \lambda+1)}, \\
D_{\lambda}^{L D}=\frac{3}{4 \pi} \frac{A m_{N} R_{0}^{2}}{\lambda}, \quad \varepsilon_{\lambda}=\hbar \sqrt{\frac{C_{\lambda}^{L D}}{D_{\lambda}^{L D}}},
\end{gathered}
$$

где $D_{\lambda}^{L D}$ - массовый параметр, $A$ - число нуклонов в ядре и $m_{N}$ - масса нуклона. Заметим, что модель жидкой капли во многих случаях (особенно для магических ядер) дает параметры поверхностных колебаний, значительно отличающиеся от экспериментальных. Для дальнейших вычислений (имея также в виду параллельные вычисления с использованием уравнений классической механики) удобно перейти к абсолютным значениям деформации ядра $s_{\lambda}=\sqrt{\frac{2 \lambda+1}{4 \pi}} R_{0} \cdot \beta_{\lambda}$. Потенциальная энергия конкретного колебания в этом случае записывается как $\frac{1}{2} c_{\lambda} s_{\lambda}^{2}$, где $c_{\lambda}=C_{\lambda} \cdot\left(\frac{2 \lambda+1}{4 \pi} R_{0}^{2}\right)^{-1}=\frac{\hbar \omega_{\lambda}}{2<s_{\lambda}^{0}>^{2}} \quad$ и $<s_{\lambda}^{0}>=\frac{R_{0}}{\sqrt{4 \pi}}<\beta_{\lambda}^{0}>$, а массовый параметр определяется из соотношения $\hbar \omega_{\lambda}=\sqrt{\frac{c_{\lambda}}{d_{\lambda}}}$, в жидкокапельной модели $d_{\lambda}^{L D}=D_{\lambda}^{L D} \cdot\left(\frac{2 \lambda+1}{4 \pi} R_{0}^{2}\right)^{-1}=\frac{3}{\lambda(2 \lambda+1)} A m_{N}$.

На рис. 2,а показан двумерный потенциал взаимодействия (3) сферического ядра ${ }^{16} \mathrm{O}$ и деформированного ядра ${ }^{154} \mathrm{Sm}\left(\beta_{2}^{\text {g.s. }}=0.3, \beta_{4}^{\text {g.s. }}=0.1\right)$ в зависимости от ориентации последнего, а на рис. 2,6 показан потенциал взаимодействия двух сферических ядер ${ }^{40} \mathrm{Ca}$ и ${ }^{90} \mathrm{Zr}$ в зависимости от их динамической квадрупольной деформации, рассчитанный с параметрами жидкокапельной модели (для простоты здесь предполагалось, что энергия деформации ядер пропорциональна их массам и вместо двух параметров динамической деформации $\beta_{1}$ и $\beta_{2}$ используется лишь один $\beta=\beta_{1}+\beta_{2}$ ). В первом случае для ядерной части взаимодействия был использован потенциал Вудса-Саксона с параметрами $V_{0}=-105$ МэВ, $r_{0}^{V}=1.12$ Фм и $a_{V}=0.75$ ФМ, а во втором случае - потенциал "proximity" с параметром $r_{0}^{i}=1.16$ Фм для радиусов ядер. Рисунки наглядно демонстрируют многомерный характер потенциала межъядерного взаимодействия и самого потенциального барьера, который, как легко видеть, невозможно характеризовать 
лишь одним значением его высоты $B$ и более правильно говорить о неком непрерывном распределении барьеров $F(B)$ (см. ниже).

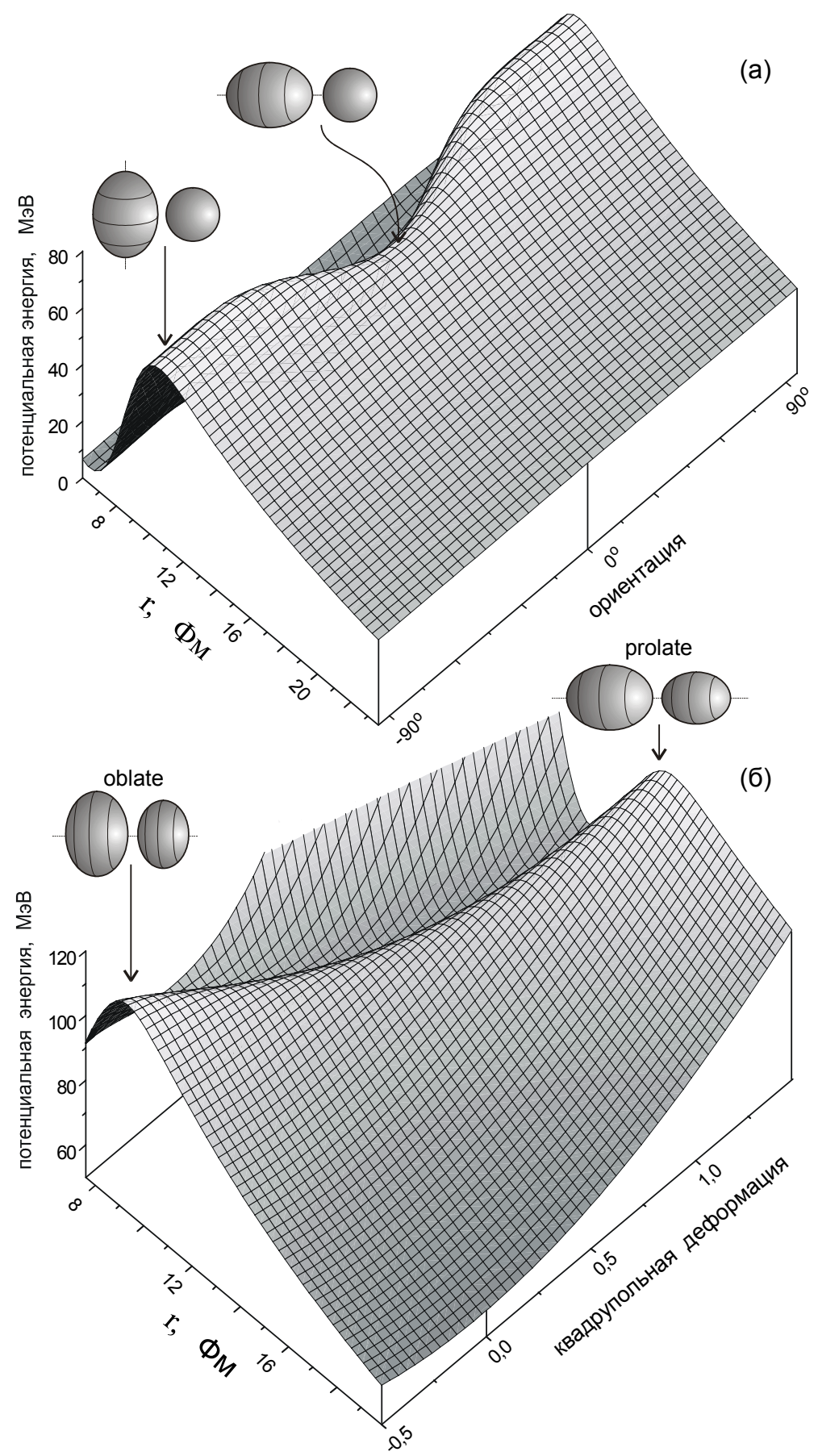

Рис. 2. (а) Потенциал взаимодействия ядер ${ }^{16} \mathrm{O}$ и ${ }^{154} \mathrm{Sm}\left(\beta_{2}^{g . s .}=0.3, \beta_{4}^{g . s .}=0.1\right)$ в зависимости от расстояния и взаимной ориентации. (б) Потенциальная энергия взаимодействия сферических ядер ${ }^{40} \mathrm{Ca}$ и ${ }^{90} \mathrm{Zr}$ в зависимости от расстояния и их динамической квадрупольной деформации 


\section{2. Система связанных уравнений и граничные условия}

Гамильтониан двух деформируемых ядер, вращающихся в плоскости реакции, записывается в виде

$$
H=-\frac{\hbar^{2} \nabla_{r}^{2}}{2 \mu}+V_{C}\left(r ; \vec{\beta}_{1}, \theta_{1}, \vec{\beta}_{2}, \theta_{2}\right)+V_{N}\left(r ; \vec{\beta}_{1}, \theta_{1}, \vec{\beta}_{2}, \theta_{2}\right)+\sum_{i=1,2} \frac{\hbar^{2} \hat{I}_{i}^{2}}{2 \mathfrak{I}_{i}}+\sum_{i=1,2} \sum_{\lambda \geq 2}\left(-\frac{1}{2 d_{i \lambda}} \frac{\partial^{2}}{\partial s_{i \lambda}^{2}}+\frac{1}{2} c_{i \lambda} s_{i \lambda}^{2}\right) .
$$

Здесь $\mu$ - приведенная масса двух ядер, а $\mathfrak{I}_{i}$ - момент инерции $i$-го ядра. В разработанном алгоритме решения квантовой системы связанных уравнений мы предполагали независимость колебаний с разной мультипольностью (не более двух в каждом ядре), а также пренебрегли связью вращения и колебаний, рассматривая их по отдельности. Как оказалось, используемый нами алгоритм дает возможность за обозримое время решать достаточно большое число связанных уравнений (порядка 300 для каждой парциальной волны на компьютере с оперативной памятью 128 Мб), что позволяет в дальнейшем отказаться от приближения независимости различных возбуждений. Максимальное число каналов, связь которых можно учесть в программе, оценивается по формуле $N_{v} \sim 500 \sqrt{M / N_{r}}$, где $N_{r}$ - число узлов сетки по радиусу $r$ и $M$ - объем оперативной памяти компьютера в мегабайтах. При решении квантовой задачи мы используем так называемое «изоцентробежное приближение» [14], заключающееся в пренебрежении собственными спинами ядер по сравнению с орбитальным моментом относительного движения, который предполагается одинаковым во всех каналах (сохраняющееся квантовое число). Это приближение позволяет значительно (в несколько раз) уменьшить размерность решаемой системы дифференциальных уравнений.

Разлагая полную волновую функцию системы по парциальным волнам:

$$
\Psi_{\overrightarrow{\mathbf{k}}}(r, \vartheta, \vec{\alpha})=\frac{1}{k r} \sum_{l=0}^{\infty} i^{l} e^{i \sigma_{l}}(2 l+1) \chi_{l}(r, \vec{\alpha}) P_{l}(\cos \vartheta)
$$

и подставляя ее в уравнение Шредингера, получим следующую систему связанных уравнений:

$$
\frac{\partial^{2}}{\partial r^{2}} \chi_{l}(r, \vec{\alpha})-\frac{l(l+1)}{r^{2}}+\frac{2 \mu}{\hbar^{2}}\left[E-V(r, \vec{\alpha})-\hat{H}_{\mathrm{int}}(\vec{\alpha})\right] \chi_{l}(r, \vec{\alpha})=0 .
$$

Здесь $\vec{\alpha}$ - внутренние переменные (параметры деформации или углы вращения), $H_{\mathrm{int}}(\vec{\alpha})$ - соответствующий этим переменным гамильтониан, $E$ - энергия сталкивающихся ядер в системе центра масс, $V(r, \vec{\alpha})=V_{C}(r, \vec{\alpha})+V_{N}(r, \vec{\alpha})$. При всех значениях $r$ за исключением области соприкосновения ядер (см. ниже) функция $\chi_{l}(r, \vec{\alpha})$ раскладывается по полному набору собственных функций гамильтониана $H_{\text {int }}(\vec{\alpha})$ :

$$
\chi_{l}(r, \vec{\alpha})=\sum_{v} y_{l, v}(r) \cdot \varphi_{v}(\vec{\alpha}),
$$

a радиальные канальные волновые функции $y_{l, v}(r)$ удовлетворяют системе обыкновенных дифференциальных уравнений второго порядка, которая в дальнейшем решается численно: 


$$
y_{l, v}^{\prime \prime}-\frac{l(l+1)}{r^{2}}+\frac{2 \mu}{\hbar^{2}}\left[E_{v}-V_{v v}(r)\right] y_{l, v}-\sum_{\mu \neq \nu} \frac{2 \mu}{\hbar^{2}} V_{\nu \mu}(r) y_{l, \mu}=0 .
$$

Здесь $E_{v}=E-\varepsilon_{v}, \quad \varepsilon_{v}-$ энергия возбуждения ядер в канале $v$, а $V_{v \mu}(r)=<\varphi_{v}|V(r, \vec{\alpha})| \varphi_{\mu}>$ - матрица связи каналов.

При низкоэнергетическом столкновении не очень тяжелых ядер, после преодоления кулоновского барьера и соприкосновения их поверхностей, ядра сливаются (т.е. образуют составное ядро) с вероятностью, близкой к единице. Сечение слияния в этом случае может быть измерено по полному выходу испарительных остатков и осколков деления составного ядра. Таким образом, при формулировке граничных условий для уравнения (14) обычно предполагается, что поток, попавший в область за кулоновским барьером, полностью поглощается, т.е. не испытывает отражения. С этой целью потребуем, чтобы при $r<R_{\text {fus }}$ функции $\chi_{l}(r, \vec{\alpha})$ имели вид сходящихся волн и не имели компонент, отвечающих волнам, отраженным от области $0 \leq r \leq R_{\text {fus }}$. В [6] в качестве $R_{\text {fus }}$ выбирается точка минимума потенциала $V_{\text {eff }}(r)=V(r, 0)+\frac{\hbar^{2}}{2 \mu} \frac{l(l+1)}{r^{2}}, \quad$ который $\quad$ при $\quad r \leq R_{f u s}$ заменяется на постоянную величину $V_{\text {eff }}\left(R_{\text {fus }}\right)$, а связь каналов в точке $r=R_{\text {fus }}$ выключается. Связанные уравнения (14) при этом расцепляются, и мы легко можем выбрать их решения, соответствующие сходящимся волнам exp $(-i q r)$. Однако резкое изменение связывающего каналы взаимодействия, величина которого достигает в этой области максимальных значений, в принципе, может приводить к дополнительному нефизическому отражению от сферы $r=R_{\text {fus }}$ и значительному искажению полной волновой функции в барьерной области.

Для устранения этого эффекта, во-первых, произведем модификацию феноменологического потенциала межъядерного взаимодействия $V(r, \vec{\alpha})$ в области перекрытия ядерных поверхностей $r \leq R_{\text {cont }}(\vec{\alpha})=R_{1}\left(\beta_{1}, \theta_{1}\right)+R_{2}\left(\beta_{2}, \theta_{2}\right)$, сделав его не зависящим от $r$ в этой области $V\left(r \leq R_{\text {cont }}, \vec{\alpha}\right)=V\left(R_{\text {cont }}, \vec{\alpha}\right)$. В качестве $R_{\text {cont }}(\vec{\alpha})$ выберем минимальное значение из двух расстояний: до точки минимума потенциала $V(r, \vec{\alpha})$ и до точки касания ядерных поверхностей. Краевую задачу для уравнений (14) будем рассматривать на интервале $\left(R_{\min }, R_{\max }\right)$, где в случае вращений $R_{\min } \leq \min \left\{R_{\text {cont }}(\vec{\alpha})\right\}, \quad$ а $\quad$ в $\quad$ случае колебаний $R_{\min } \leq R_{\text {cont }}\left(-\sqrt{M_{1}}<\vec{\beta}_{1}^{0}>,-\sqrt{M_{2}}<\vec{\beta}_{2}^{0}>\right)$, где $M_{1}, M_{2} \quad$ - максимальные числа учитываемых каналов, $\left.\left\langle\vec{\beta}_{1}^{0}\right\rangle,<\vec{\beta}_{2}^{0}\right\rangle$ - вектора среднеквадратичных значений параметров деформации, а $R_{\max }>R_{1}+R_{2}$. Центробежный потенциал также заменим на постоянное значение $\frac{\hbar^{2}}{2 \mu} \frac{l(l+1)}{R_{s}^{2}}$ при $r<R_{s}$, где в качестве $R_{s}>R_{\min }$ мы выбираем минимальное из двух расстояний: точки минимума эффективного потенциала сферических ядер $V_{\text {eff }}(r, 0)$ и точки касания сферических ядер. Выбор 
$R_{\min }$ достаточно произволен. Важно, чтобы эта величина лежала в области за кулоновским барьером $R_{\min }<R_{B}(\vec{\alpha})$. Во-вторых, при формулировке граничных условий в точке $r=R_{\text {fus }}=R_{s} \leq R_{\text {cont }}$, с которой начинается интегрирование системы дифференциальных уравнений (14), воспользуемся точным решением этих уравнений в области $r \leq R_{\text {fus }}$, которое может быть легко найдено в случае постоянства всех коэффициентов уравнений.

Выполним при $r \leq R_{\text {fus }}$ линейное преобразование

$$
y_{l, v}(r)=\sum_{v} Y_{l, n}(r) A_{n, v},
$$

диагонализующее матрицу $W_{v \mu}=V_{v \mu}\left(R_{f u s}\right)+\varepsilon_{v} \delta_{v \mu}$, так что $\left\{A^{-1} W A\right\}_{n m}=\tilde{W}_{n n} \delta_{n m}$. Новые функции $Y_{l, n}(r)$ удовлетворяют независимым уравнениям

$$
Y_{l, n}^{\prime \prime}+K_{l, n}^{2} Y_{l, n}=0, \quad K_{l, n}^{2}=\frac{2 \mu}{\hbar^{2}}\left[E-\tilde{\varepsilon}_{n}-\tilde{U}_{0}-U_{l}\right] .
$$

Потоку внутрь ядра в открытых каналах соответствуют частные решения этих уравнений $\quad Y_{l, n}(r)=N_{l, n} \exp \left(-i K_{l, n} r\right), \quad$ для $\quad$ которых $\quad Y_{l, n}^{\prime}(r)=-i K_{l, n} Y_{l, n}(r) . \quad$ Из линейного преобразования (15) следуют граничные условия для искомых канальных волновых функций $y_{l, v}(r)$ при $r \leq R_{\text {fus }}$

$$
y_{l, v}^{\prime}(r)=\sum_{n} Y_{l, n}^{\prime}(r) A_{n, v}=\sum_{\mu} C_{v \mu} y_{l, \mu}(r),
$$

где $C_{v \mu}=-i \sum_{n}\left(A^{-1}\right)_{n, v} K_{l, n} A_{n, v}$. Значения матрицы $\tilde{W}_{n n}$ представляют собой собственные значения матрицы $W_{\nu \mu}$, а матрица $A_{n v}$ составлена из ее нормированных собственных векторов. Практическое их нахождение может быть выполнено так называемым QR-методом [15].

На больших расстояниях для волновой функции имеем стандартные граничные условия в виде падающей и расходящейся волны в упругом канале $v=0$ и расходящихся волн в остальных каналах. Для парциальных волновых функций открытых каналов это соответствует условию

$$
y_{l, v}(r \rightarrow \infty)=\frac{i}{2}\left[h_{l}^{(-)}\left(\eta_{v}, k_{v} r\right) \cdot \delta_{v 0}-\left(\frac{k_{0}}{k_{v}}\right)^{1 / 2} S_{v 0}^{l} \cdot h_{l}^{(+)}\left(\eta_{v}, k_{v} r\right)\right],
$$

где $k_{v}^{2}=\frac{2 \mu}{\hbar^{2}} E_{v}, \eta_{v}=\frac{k_{v} Z_{1} Z_{2} e^{2}}{2 E_{v}}$ - параметр Зоммерфельда, $\sigma_{l, v}=\arg \Gamma\left(l+1+i \eta_{v}\right)$ кулоновская фаза, $h_{l}^{( \pm)}\left(\eta_{v}, k_{v} r\right)$ - кулоновские парциальные волновые функции с асимптотикой $\exp \left( \pm i x_{l, v}\right), \quad x_{l, v}=k_{v} r-\eta_{v} \ln 2 k_{v} r+\sigma_{l, v}-l \pi / 2, \quad S_{v 0}^{l}-$ парциальная матрица рассеяния. Исключая в (19) неизвестные величины $S_{v 0}^{l}$, получаем на больших расстояниях граничные условия третьего рода

$$
\left[y_{l, v} \frac{d h_{l}^{(+)}}{d r}-\frac{d y_{l, v}}{d r} h_{l}^{(+)}\right]_{r=R_{\max }}=k_{0} \delta_{v 0}
$$


которых вместе с условиями (17) достаточно для численного решения системы связанных дифференциальных уравнений второго порядка (14). Для закрытых каналов $\left(E_{v}<0\right)$ получаются аналогичные выражения с кулоновскими функциями мнимого аргумента.

В конкретных расчетах граничные условия (18) используются фактически при некотором конечном радиусе $R_{\max } \sim 30 \div 40$ Фм. Для очень тяжелых ядер с большим значением $Z$ наличие слабо убывающего $\left(\sim r^{-3}\right)$ связывающего каналы кулоновского взаимодействия (4) приводит к тому, что при $r=R_{\max }$ ядра уже находятся в возбужденном состоянии, и мы не имеем права использовать граничные условия (19) в этой точке. Физический смысл более правильных граничных условий достаточно очевиден. Кулоновское отталкивание приводит к отрицательным динамическим деформациям (сплюснутые ядра) и к более предпочтительной ориентации «бок к боку» вращающихся квадрупольно деформированных ядер. Существует два способа количественного решения этой проблемы. В области $r \geq R_{\max }$ можно ограничиться меньшим числом связанных каналов и решить численно задачу на интервале $R_{\max } \leq r \leq R_{M A X}$, например, в приближении слабой связи каналов. Выбрав $R_{\text {мAX }}$ порядка 300 Фм и установив при $r \leq R_{\max }$ «безотражательные» граничные условия, можно найти в этой точке значения всех канальных волновых функций, которые в дальнейшем должны быть использованы вместо граничных условий (19) при численном решении полной системы уравнений (14) на интервале $R_{\text {fus }} \leq r \leq R_{\max }$. Другим методом нахождения более правильных граничных условий в точке $r=R_{\max }$ является аналитическое решение задачи в адиабатическом приближении, которое предполагает наиболее вероятным движение системы вдоль дна многомерной потенциальной поверхности. В этом случае мы можем найти основное состояние системы для гамильтониана $H_{\mathrm{int}}(\vec{\alpha})+V\left(R_{\max }, \vec{\alpha}\right)$, а затем разложить это состояние по функциям $\varphi_{v}(\vec{\alpha})$. Найденные коэффициенты разложения дадут нам амплитуды сходящихся волн при $r \leq R_{\max }$ во всех каналах, а не только в канале $v=0$, как записано в соотношении (18). Анализ эффектов дальнодействующего кулоновского возбуждения в процессах околобарьерного слияния ядер будет опубликован нами отдельно.

Связывающее каналы взаимодействие $V(r, \vec{\alpha})$ может быть разделено на слабо убывающую кулоновскую часть (4) и быстро убывающую ядерную часть, и, соответственно, матрица связи каналов $V_{v \mu}(r)=V_{v \mu}^{C}(r)+V_{v \mu}^{N}(r)$. Кулоновская часть матрицы связи каналов известна в явном виде (см., например, [6]). Для того, чтобы учесть в явном виде нелинейные эффекты ядерного взаимодействия, при вычислении матричных элементов $V_{\nu \mu}^{N}(r)$ в [6] применены методы матричной алгебры. Фактически такой подход эквивалентен разложению функции $V^{N}(r, \vec{\alpha})$ в ряд по степеням $\vec{\alpha}$ до $M$-го порядка, где $M$ - максимальный номер учитываемых возбужденных состояний. В данной работе величины $V_{v \mu}^{N}(r)$ при каждом значении 
$r$ вычисляются в явном виде с помощью квадратурных формул Гаусса (для вращений) и Гаусса-Эрмита (для колебаний) порядка $N$. При сохранении в функции $V^{N}(r, \vec{\alpha})$ членов разложения по степеням $\vec{\alpha}$ до $k$-го порядка, для получения точного значения матричных элементов достаточно использовать порядок $N \geq M+(k+1) / 2$ по каждой степени свободы. Наш опыт показывает, что выбор $M=10$ для вращений и $M=6$ для колебаний достаточен для получения вполне аккуратного результата при использовании реалистических межъядерных взаимодействий.

Сечение слияния определяется отношением поглощенного потока к падающему

$$
\sigma_{f u s}(E)=\frac{\pi}{k_{0}^{2}} \sum_{l=0}^{\infty}(2 l+1) \cdot T_{l}(E),
$$

где $T_{l}(E)$ - парциальные коэффициенты барьерной проницаемости

$$
T_{l}(E)=\sum_{v} \frac{j_{l, v}}{j_{0}} .
$$

Здесь $j_{l, v}=-\left.i \frac{\hbar}{2 \mu}\left(y_{l, v} \frac{d y_{l, v}^{*}}{d r}-y_{l, v}^{*} \frac{d y_{l, v}}{d r}\right)\right|_{r \leq R_{f t s}}$ - парциальный поток в канале $v, \mathbf{a}$ $j_{0}=\hbar k_{0} / \mu$. При слиянии более тяжелых ядер (особенно для симметричных комбинаций) вероятность образования составного ядра после соприкосновения ядерных поверхностей оказывается меньше единицы за счет процессов квазиделения [16]. Вычисление этой вероятности чрезвычайно сложно [17] и представляет самостоятельную задачу, выходящую за рамки рассматриваемого здесь алгоритма. Для таких систем рассчитанное по формуле (20) сечение будет соответствовать так называемому сечению захвата, равному сумме сечений слияния и квазиделения.

\section{4. Численное решение системы связанных уравнений}

Система обыкновенных дифференциальных уравнений (14) для конечного числа канальных функций с граничными условиями (18), (19) образует смешанную краевую задачу. Для ее численного решения вводится сетка, в узлах сетки определяются сеточные функции

$$
r_{j}=r_{0}+j h, \quad y_{l, v}^{j}=y_{l, v}\left(r_{j}\right), j=0,1, \ldots, J, r_{0}=R_{f u s}-2 h .
$$

Граничные условия, содержащие первую производную, аппроксимируются двухточечной разностной схемой. Условие (19) для открытых каналов приводит к соотношению

$$
\begin{aligned}
& y_{l, v}\left(r_{J}\right)=\tau y_{l, v}\left(r_{J-1}\right)-\zeta \\
& \tau=\frac{2+h \gamma}{2-h \gamma}, \quad \zeta=\frac{2 h k_{v} \delta_{v 0}}{(2-h \gamma) h_{l}^{(+)}\left(\eta_{v}, k_{v} r_{J-1 / 2}\right)}, \quad \gamma=\frac{d h_{l}^{(+)}\left(\eta_{v}, k_{v} r_{J-1 / 2}\right) / d r}{h_{l}^{(+)}\left(\eta_{v}, k_{v} r_{J-1 / 2}\right)} .
\end{aligned}
$$

Аналогичные формулы получаются и для закрытых каналов. Граничное условие (17) дает матричное соотношение 


$$
\begin{gathered}
y_{l, v}\left(r_{0}\right)=\sum_{\mu} \Theta_{v \mu} y_{l, \mu}\left(r_{1}\right), \\
\Theta=D^{-1} F, \quad D_{v \mu}=\delta_{v \mu}+\frac{h}{2} C_{v \mu}, \quad F_{v \mu}=\delta_{v \mu}-\frac{h}{2} C_{v \mu} .
\end{gathered}
$$

Для аппроксимации дифференциальных уравнений разностными обычно используется хорошо зарекомендовавший себя метод Нумерова (см., например, [18]), основанный на трехточечной аппроксимации второй производной и обеспечивающий фактически порядок аппроксимации $O\left(h^{4}\right)$ на одном шаге при решении уравнения Шредингера вдоль сетки. Этот метод используется и в работе [6]. Разностные уравнения, полученные записью дифференциальных уравнений (14) во внутренних узлах сетки, в совокупности с граничными условиями (23) и (24) для пар крайних узлов образуют систему линейных уравнений, матрица которой является ленточной. Для уменьшения числа ненулевых элементов матрицы при аппроксимации второй производной вместо метода Нумерова мы используем трехточечную разностную схему

$$
y^{\prime \prime}\left(r_{j}\right)=h^{-2}\left(y_{j-1}-2 y_{j}+y_{j+1}\right)+O\left(h^{2}\right),
$$

при этом полуширина матрицы оказывается примерно вдвое меньше, чем для метода Нумерова. Хотя схема (25) при решении уравнения Шредингера обеспечивает порядок аппроксимации $O\left(h^{2}\right)$, этого вполне достаточно для получения решения с хорошей точностью. Отметим, что погрешность численного решения краевой задачи (14) возникает и накапливается не только из-за разностной аппроксимации производных, но также и при решении системы линейных уравнений. При $h \rightarrow 0$ первая составляющая этой погрешности уменьшается, а вторая растет из-за возрастания числа уравнений и вычислительных операций. В результате при уменьшении шага общая погрешность вначале уменьшается, а затем нарастает. Тестирование двух алгоритмов показало, что точность самой схемы Нумерова оказывается, как правило, избыточной, а при использовании многоходового алгоритма решения краевой задачи его результирующая погрешность может превышать погрешность метода, основанного на схеме (25).

Поскольку современные компьютеры вполне позволяют сохранять в оперативной памяти всю матрицу в целом, мы используем метод Гаусса для непосредственного решения системы линейных уравнений и нахождения значений $y_{l, v}\left(r_{j}\right)$ во всех узлах сетки. Основным преимуществом подхода на основе метода Гаусса является его большая устойчивость в расчетах с конечной разрядностью. Схема с одним прямым и одним обратным ходом представляется предпочтительнее, чем схема с несколькими проходами в одном направлении и последующим решением системы линейных уравнений для определения произвольных постоянных. В последнем случае возможно нарастание погрешности из-за утраты необходимой точности убывающего решения в классически недоступной области, что особенно важно при решении задач с двумя точками поворота. Использование метода Гаусса для непосредственного решения системы линейных уравнений позволило увеличить почти на порядок число учитываемых связанных каналов по сравнению с возможностью, реализованной в 
данный момент в программе CCFULL [6]. Это особенно важно при возбуждении нескольких степеней свободы в обоих ядрах, а также для избежания нефизических эффектов девозбуждения ядер, связанных с резким ограничением числа учитываемых каналов. Хранение в памяти всех значений $y_{l, v}\left(r_{j}\right)$ позволяет также легко восстановить и сами многоканальные волновые функции (13) и (11) и, следовательно, получить дополнительное, более наглядное представление о динамике проницаемости многомерного потенциального барьера (см. ниже).

\section{4. Функция распределения по барьерам и полуэмпирическая модель слияния ядер}

Проводимые в последнее время прецизионные эксперименты по измерению энергетической зависимости сечения околобарьерного слияния позволяют достаточно аккуратно оценить вторую производную по энергии от величины $E \sigma_{\text {fus }}(E)$, которая в классическом пределе может быть отождествлена с так называемой «функцией распределения по барьерам» [20]

$$
D(B)=\frac{1}{\pi R_{B}^{2}} d^{2}\left(E \sigma_{f u s}\right) /\left.d E^{2}\right|_{E=B} .
$$

Основным итогом таких измерений является обнаружение довольно сложной структуры функции $D(B)$ в околобарьерной области энергий [2-4] (различной для различных пар ядер), что свидетельствует о сложной (нетривиальной) динамике прохождения потенциального барьера в условиях сильной связи каналов.

В отсутствие связи каналов (пренебрежение всеми степенями свободы, кроме относительного движения сферических ядер) эффективный потенциал взаимодействия $V_{\text {eff }}(r)=V(r)+\frac{\hbar^{2}}{2 \mu} \frac{l(l+1)}{r^{2}}$ можно аппроксимировать вблизи его максимума «перевернутой» параболой $V_{\text {eff }}(r) \approx B(l)+1 /\left.2 V^{\prime \prime}(r)\right|_{r=R_{B}}\left[r-R_{B}(l)\right]^{2}$. В этом случае проницаемость барьера определяется хорошо известной формулой Хилла-Уилера [21]

$$
T(l, E)=\left[1+\exp \left(\frac{2 \pi}{\hbar \omega_{B}}[B(l)-E]\right)\right]^{-1},
$$

где $B(l), R_{B}(l)$ и $\omega_{B}(l)=\sqrt{-V^{\prime \prime}\left(R_{B}\right) / \mu}$ - высота, положение и осцилляторная частота барьера, характеризующая его ширину и, вообще говоря, зависящая от $E$. Если теперь предположить, что положение эффективного барьера $R_{B}(l)$ слабо изменяется с изменением $l$, то высота барьера определяется выражением $B(l)=B+\frac{\hbar^{2}}{2 \mu R_{B}^{2}} l(l+1)$, где $B$ и $R_{B}$ - высота и положение барьера при $l=0$. В этом случае проницаемость $T(l, E)$ зависит от $B$ и от $l$ не произвольно, а 
является функцией аргумента $\quad x=B+\frac{\hbar^{2}}{2 \mu R_{B}^{2}} l(l+1)-E, \quad$ т.е. $\quad T(l, E)=f(x)$. Используя выражение (20) для сечения слияния, имеем

$$
\frac{d\left(E \sigma_{f u s}\right)}{d E}=\frac{\pi \hbar^{2}}{2 \mu} \sum_{l=0}^{\infty}(2 l+1) \frac{d T(l, E)}{d E} .
$$

Поскольку $\frac{d T}{d E}=-\frac{d T}{d x}=-\frac{d T}{d l}\left(\frac{d x}{d l}\right)^{-1}=-\frac{d T}{d l} \frac{2 \mu R_{B}^{2}}{\hbar^{2}} \frac{1}{2 l+1}$, то $\frac{d\left(E \sigma_{f u s}\right)}{d E}=-\pi R_{B}^{2} \sum_{l=0}^{\infty} \frac{d T(l, E)}{d l}$. При столкновении тяжелых ядер, когда в сечение большой вклад вносят много парциальных волн, $T(l, E)$ является гладкой функцией $l$ и суммирование в (28) может быть заменено на интеграл по $l$, который легко вычисляется, давая $d\left(E \sigma_{\text {fus }}\right) / d E=\pi R_{B}^{2} \cdot T(l=0, E)$, или

$$
D(E)=\frac{1}{\pi R_{B}^{2}} \frac{d^{2}\left(E \sigma_{f u s}\right)}{d E^{2}}=\frac{d T(l=0, E)}{d E} .
$$

В классическом случае $T(E)=1$ при $E>B \quad$ и $T(E)=0$ при $E<B$, т.е. $D(E)=\delta(E-B)$. В квантовом случае проницаемость одномерного барьера определяется выражением (27) и функция $D(E)$ имеет один максимум при $E=B$ с шириной $\Delta_{B}=\hbar \omega_{B} \ln (17+12 \sqrt{2}) / 2 \pi \approx 0.56 \hbar \omega_{B}$ (для параболического барьера).

В реальном случае потенциал ядро-ядерного взаимодействия является многомерной функцией (см. рис. 2) и падающий поток преодолевает кулоновский барьер в разных точках, т.е. при разных значениях $B$ (разных значениях динамической деформации и/или ориентации ядер). Для простой оценки проницаемости такого многомерного барьера была предложена полуэмпирическая модель [17], основанная на использовании параметризованной функции распределения по барьерам. В этом подходе полная проницаемость усредняется по высоте барьера $B$ и вместо (27) имеем

$$
T(l, E)=\int F(B)\left[1+\exp \left(\frac{2 \pi}{\hbar \omega_{B}}\left[B+\frac{\hbar^{2}}{2 \mu R_{B}^{2}(l)} l(l+1)-E\right]\right)\right]^{-1} d B,
$$

где функция $F(B)$ удовлетворяет условию нормировки $\int F(B) d B=1$ и может быть аппроксимирована симметричным гауссианом с центром в $B_{0}=\left(B_{1}+B_{2}\right) / 2$ и шириной $\Delta_{B}=\left(B_{2}-B_{1}\right) / 2$. Для статически деформированных ядер величины $B_{1}$ и $B_{2}$ определяются как барьеры двух предельных конфигураций «нос к носу» и «бок к боку» (рис. 2,a). В этом случае может быть использован и прямой метод усреднения по ориентациям ядер с нахождением реального барьера $B\left(\vec{\beta}_{1}, \theta_{1} ; \vec{\beta}_{2}, \theta_{2}\right)$. Для ядер с нулевой статической деформацией $B_{1}$ соответствует минимальному значению многомерного барьера с учетом динамической деформации (седловая точка на рис. 2,б), а $B_{2}$ - барьеру взаимодействия сферических ядер. Наш опыт показывает, что для более аккуратного описания сечения слияния очень тяжелых ядер, когда разность $\left(B_{2}-B_{1}\right)$ велика, функцию 
$F(B)$ лучше аппроксимировать слегка асимметричным гауссианом с меньшей «внутренней» полушириной (при меньших значениях $B$ ) [17].

\section{5. Сечения слияния ядер при наличии сильной связи каналов}

Для того, чтобы выполнить сравнение рассчитанных сечений слияния ядер не только с экспериментальными данными, но и с результатами расчетов по программе CCFULL [6], мы используем здесь для межъядерного взаимодействия потенциал Вудса-Саксона, поскольку программа CCFULL не имеет возможности работать с потенциалом "proximity".
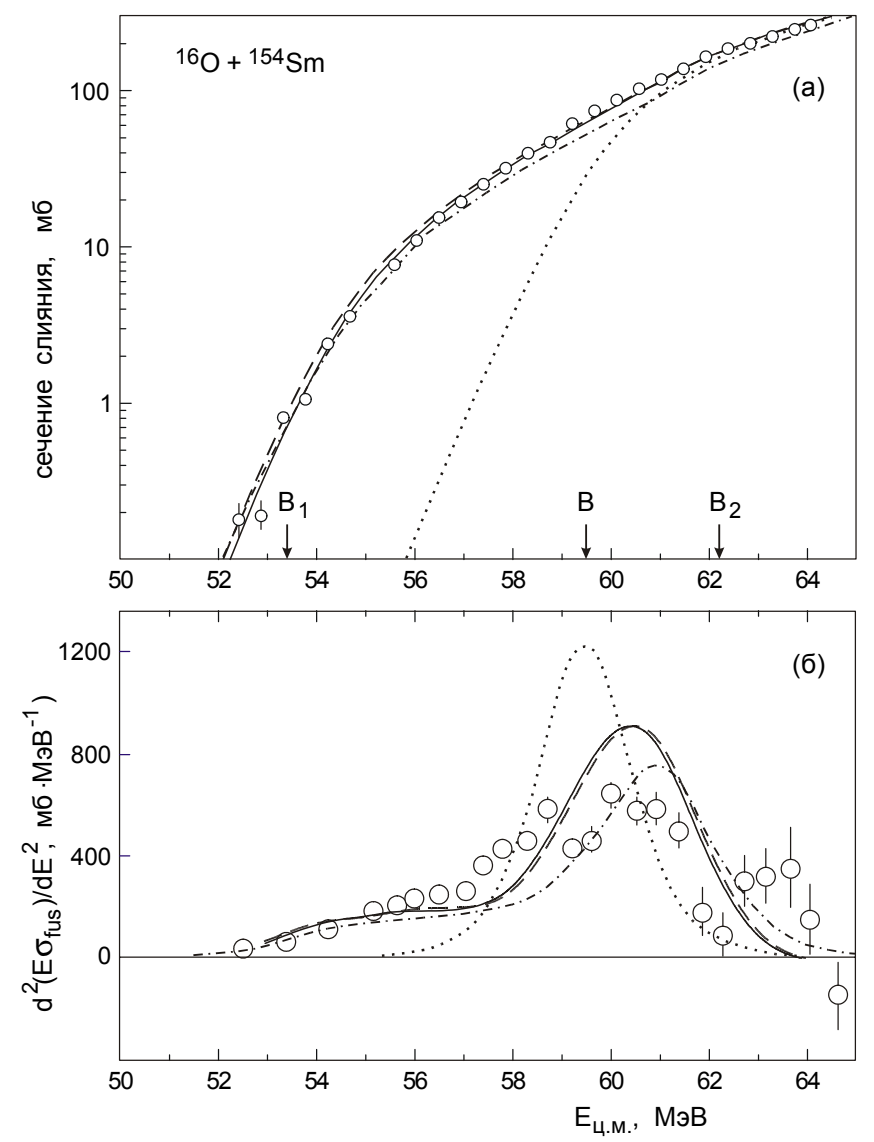

Рис. 3. Сечение слияния ядер кислорода с деформированным ядром ${ }^{154} \mathrm{Sm}$ (а) и «функция распределения по барьерам» (б). Точечная кривая - слияние сферических ядер, пунктирная и сплошная кривые соответствуют расчетам по программе CCFULL [6] и по нашей программе с учетом возбуждения 5 ротационных состояний ядра ${ }^{154} \mathrm{Sm}$. Штрихпунктирная кривая показывает сечение, полученное простым усреднением по ориентациям деформированного ядра. Экспериментальные данные взяты из [19]. Стрелками указаны положения кулоновских барьеров для сферических ядер и для двух предельных ориентаций деформированного ядра-мишени

При расчете сечения слияния ядра ${ }^{16} \mathrm{O}$ с деформированным ядром ${ }^{154} \mathrm{Sm}$ были использованы следующие параметры: $V_{0}=-105 \mathrm{MэB}, r_{0}^{V}=1.12$ Фм, 
$a_{V}=0.75$ Фм (см. рис. 2,a), $r_{0}^{1}=1.2$ ФМ, $r_{0}^{2}=1.06$ ФМ, параметры статической квадрупольной и гексадекапольной деформации ядра ${ }^{154} \mathrm{Sm}$ соответственно $\beta_{2}=0.3$ и $\beta_{4}=0.1$, энергия первого возбужденного ротационного уровня $E_{2+}=0.084$ МэВ,$R_{\max }=24$ ФМ и $h=0.05$ ФМ. На рис. 3 показаны сечения слияния, рассчитанные с учетом возбуждения пяти ротационных состояний по нашей программе и по программе CCFULL, в сравнении экспериментальными данными [19]. На этом же рисунке показана и «функция распределения по барьерам» $d^{2}\left(E \sigma_{f u s}\right) / d E^{2}$, позволяющая более детально разглядеть «тонкую структуру» и сложный характер барьерной проницаемости. Как видно из рисунка, для данной реакции обе программы дают практически тождественные значения. Заметим, что при слиянии деформированных ядер правильный выбор межъядерного потенциала взаимодействия в сочетании с простым усреднением одномерных барьерных проницаемостей по ориентациям ядер (т.е. фактически по высоте барьера, см. рис. 2,a) также приводит к вполне удовлетворительному согласию с экспериментом как по величине сечения слияния в подбарьерной области, так и по форме функции распределения по барьерам.

На рис. 4,a показаны сечения слияния сферических ядер ${ }^{36} \mathrm{~S}$ и ${ }^{90} \mathrm{Zr}$, рассчитанные $\mathrm{c}$ учетом возбуждения 4 фононов октупольных колебаний поверхности ядра ${ }^{90} \mathrm{Zr}\left(\lambda=3, \hbar \omega_{\lambda}=2.75 \mathrm{MэB}, \quad<\beta_{\lambda}^{0}>=0.22\right)$, а на рис. 4,6 приведены соответствующие функции распределения по барьерам. Экспериментальные данные взяты из работы [22]. Ядро-ядерное взаимодействие сферических ядер в этом случае также было выбрано в форме потенциала ВудсаСаксона с глубиной $V_{0}=-77.5$ МэВ, радиусом $r_{0}=1.15$ Фм и диффузностью $a=0.8$ Фм. Этот потенциал дает значение кулоновского барьера $B_{0}=-78.3$ МэВ (правая стрелка на рис. 4,a). Учет октупольных колебаний ядра-мишени значительно увеличивает сечение слияния в подбарьерной области, при этом обе программы (CCFULL [6] и наша) также дают очень близкие результаты.

Увеличение сечения слияния в «подбарьерной» области $E<B_{0}$ легко объясняется c помощью рис. 2,б. Динамическая деформация поверхности приводит к понижению кулоновского барьера для случая вытянутой конфигурации (положительные значения $\beta_{\lambda}$ ). При дальнейшем увеличении деформации потенциальная энергия вновь увеличивается из-за ненулевой жесткости ядерной поверхности $C_{\lambda}$. Таким образом, в суммарном ядро-ядерном потенциале можно выделить седловую точку $\left(r_{s d}, \beta_{s d}\right)$, соответствующую минимальному значению $B_{s d}$ кулоновского барьера в пространстве $(r, \beta)$ (см. рис. 2,б). Используя экспериментальное значение энергии фонона октупольных колебаний ядра ${ }^{90} \mathrm{Zr}\left(\hbar \omega_{\lambda=3}=2.75 \mathrm{MэB}\right)$, вычислив жесткость соответствующего осциллятора (см. раздел 1) и построив двумерную поверхность ядро-ядерного взаимодействия (аналогичную той, что показана на рис. 2,б), мы легко можем найти для данного случая значение кулоновского барьера в седловой точке $B_{s d}=75.6$ МэВ (левая стрелка на рис. 4,a). Предполагая, что падающий поток, 
двигаясь в пространстве $(r, \beta)$, проходит двумерный барьер при различных значениях динамической деформации в интервале $\left(0 \leq \beta<\beta_{s d}\right)$, можно аппроксимировать функцию распределения по барьерам гауссианом с центром в точке $\left(B_{0}+B_{s d}\right) / 2$ и полушириной $\left(B_{0}-B_{s d}\right) / 2$, а затем вычислить и полное сечение слияния. Полученное в таком полуэмпирическом подходе сечение вполне удовлетворительно согласуется с экспериментом и с точным расчетом (см. штрихпунктирные кривые на рис. 4), давая вместе с тем достаточно наглядное понимание увеличения подбарьерной проницаемости и уширения функции распределения по барьерам.
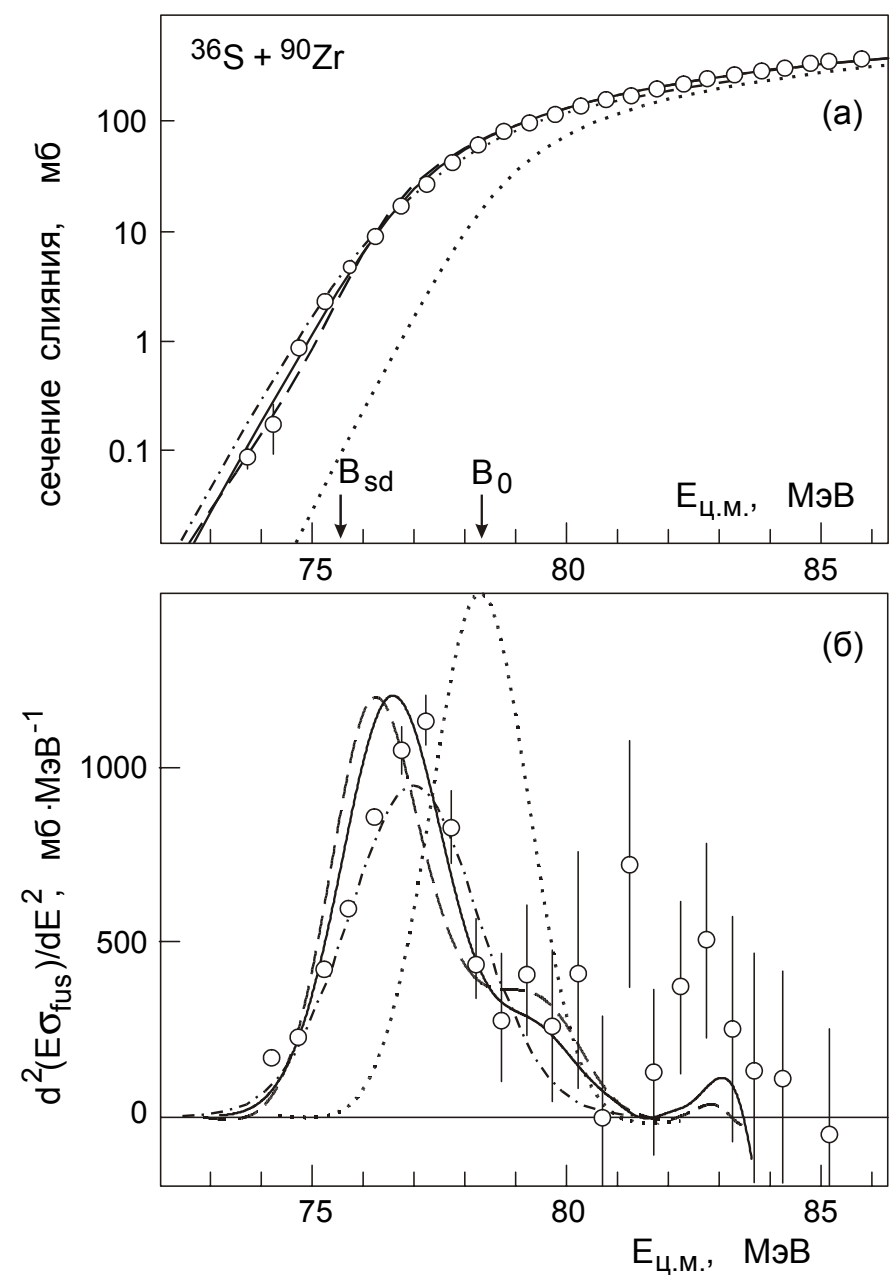

Рис. 4. Сечение слияния сферических ядер ${ }^{36} \mathrm{~S}$ и ${ }^{90} \mathrm{Zr}$ (а) и «функция распределения по барьерам» (б). Точечная кривая - расчет без учета динамических деформаций, пунктирная и сплошная кривые соответствуют расчетам по программе CCFULL [6] и по нашей программе с учетом возбуждения 4 фононов октупольных колебаний поверхности ядра ${ }^{90} \mathrm{Zr}$. Штрихпунктирная кривая показывает сечение, полученное с помощью эмпирической модели учета динамических деформаций (см. текст). Экспериментальные данные взяты из работы [22]

Подтверждение этой качественной картины можно видеть также на рис. 5, где показан модуль двумерной волновой функции относительного движения ядер 
${ }^{36} \mathrm{~S}$ и ${ }^{90} \mathrm{Zr}$ в пространстве $\left(r, s_{\lambda=3}\right)$, где $s_{\lambda=3}=\sqrt{(2 \lambda+1) / 4 \pi} R_{0} \beta_{\lambda=3}$ - абсолютное значение октупольной деформации ядра ${ }^{90} \mathrm{Zr}$. Видно, что на больших расстояниях многоканальная волновая функция сконцентрирована в области малых деформаций $\beta_{3} \approx 0$, что отражает доминирование нулевых колебаний основного состояния ядра $\varphi_{v=0}(\beta)$ в разложении (13). При низких энергиях (медленные столкновения) ядра испытывают заметную деформацию в момент соприкосновения. При этом кулоновский барьер преодолевается преимущественно при положительных значениях деформации (вытягивание ядер навстречу друг другу), приводящих к понижению высоты этого барьера (см. рис. 2,a и ландшафт потенциальной энергии на рис. 5). Осцилляции модуля волновой функции на больших расстояниях являются результатом интерференции падающей и отраженной волн.
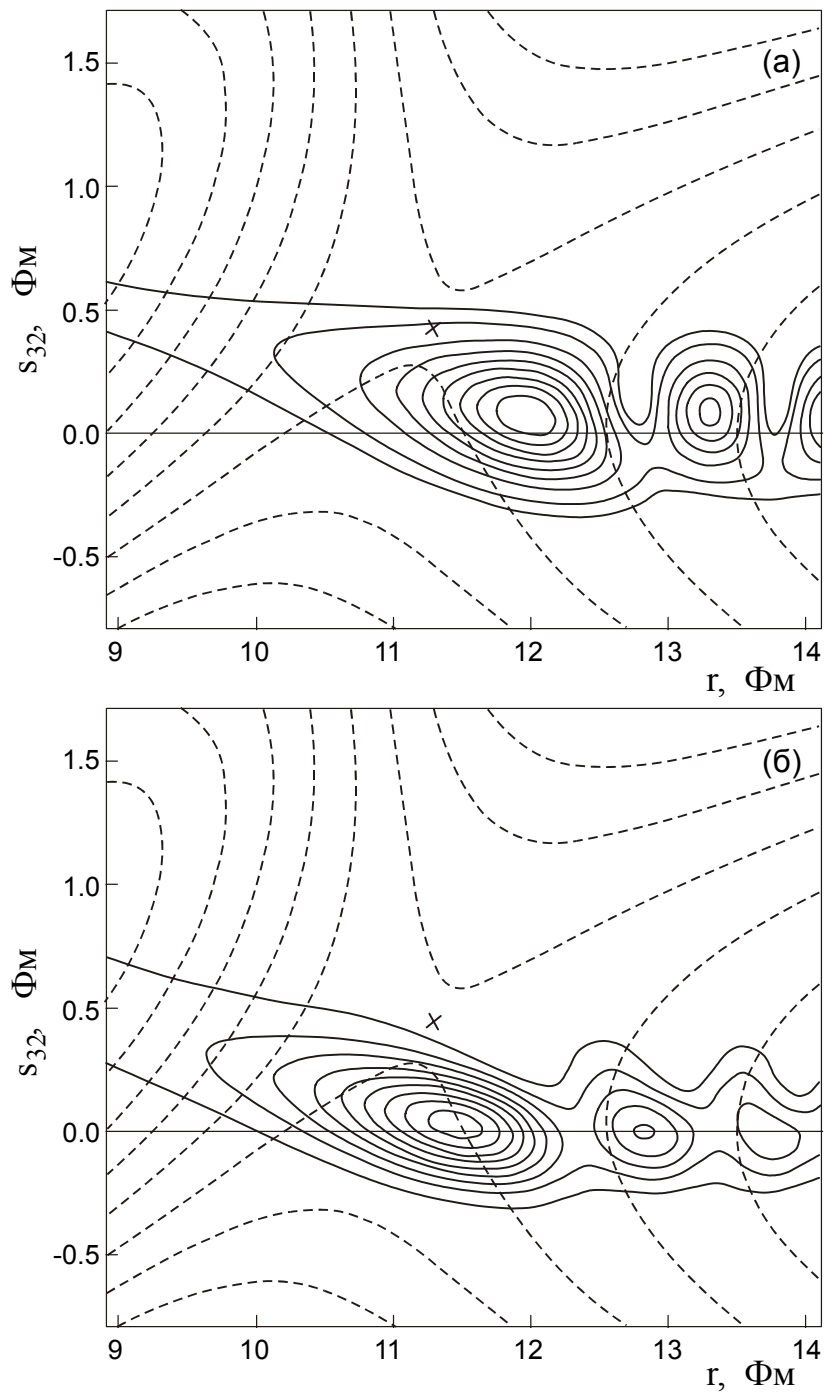

Рис. 5. Топографический ландшафт квадрата модуля двумерной волновой функции (13), описывающей процесс слияния ядер ${ }^{36} \mathrm{~S}$ и ${ }^{90} \mathrm{Zr}$ при $l=0$ (сплошные линии), на фоне ландшафта потенциальной энергии (пунктир) при энергиях $E_{c m}=77$ МэВ (а) и $E_{c m}=80 \mathrm{MэB} \mathrm{(б).} \mathrm{Крестиком} \mathrm{обозначено} \mathrm{положение} \mathrm{седловой} \mathrm{точки} \mathrm{кулоновского} \mathrm{барьера}$ 


\section{6. Синтез сверхтяжелых ядер}

При слиянии очень тяжелых ядер, используемых, в частности, для синтеза сверхтяжелых элементов, связь каналов играет еще более заметную роль. Для этой области ядер имеется гораздо меньше экспериментальных данных по сечениям слияния, а для случая более или менее симметричных комбинаций они вообще не могут быть получены из-за невозможности разделения продуктов процессов глубоконеупругого рассеяния и процесса деления составного ядра. Это значительно усиливает важность теоретических расчетов и предсказаний в этой области ядер. Надежность таких предсказаний сегодня не очень высока, что объясняется не только сложностью учета связи нескольких степеней свободы в процессе околобарьерного слияния, но также и неточностью в определении ядроядерного взаимодействия. До последнего времени не было возможности проводить квантовые расчеты процесса слияния очень тяжелых ядер в рамках метода связанных каналов, поскольку существующие алгоритмы (и в частности программа CCFULL) работают в этом случае довольно неустойчиво. Как оказалось, разработанный нами новый алгоритм решения системы большого числа связанных уравнений позволяет проводить такие расчеты.

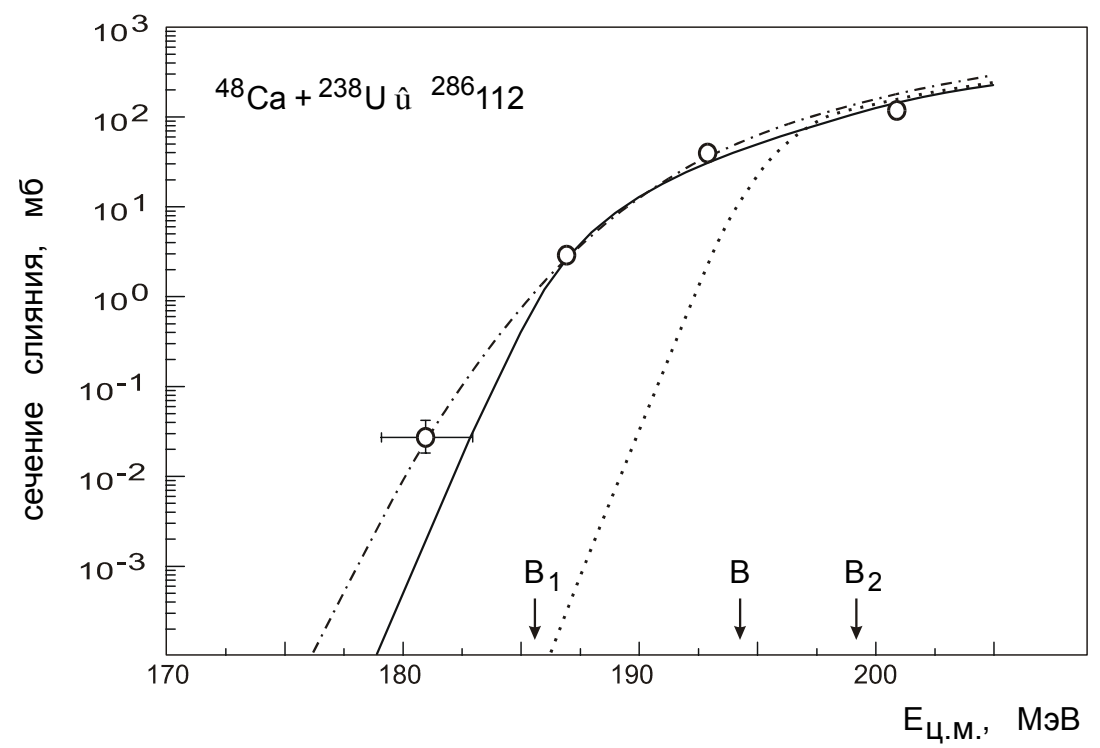

Pис. 6. Сечение слияния ядер ${ }^{48} \mathrm{Ca}$ с деформированным ядром ${ }^{238} \mathrm{U}$. Точечная кривая - слияние сферических ядер. Сплошная кривая соответствует расчету по методу связанных каналов с использованием предлагаемого нами алгоритма. Штрихпунктирная кривая показывает сечение, полученное в полуэмпирическом подходе. Экспериментальные данные взяты из [23]. Стрелками указаны положения кулоновских барьеров для сферических ядер (барьер Басса) и для двух предельных ориентаций деформированного ядра-мишени

На рис. 6 показаны экспериментальные и теоретические сечения слияния ядер ${ }^{48} \mathrm{Ca}{ }^{238} \mathrm{U}$. Экспериментальные данные, взятые в работе [23], соответствуют выходу всех продуктов деления (обычного и быстрого, идущего без образования составного ядра), поэтому в данном случае более правильно говорить о сечении захвата (см. комментарий в конце параграфа 2). Для ядерного взаимодействия мы 
опять выбрали потенциал Вудса-Саксона с параметрами $V_{0}=-160$ МэВ, $r_{0}^{V}=1.14$ Фм и $a_{V}=0.65$ Фм, обеспечивающий высоту кулоновского барьера $B=194$ МэВ, предсказываемую моделью Басса [24] для этих ядер. Статическая квадрупольная деформация $\beta_{2}=0.215$ [25] ядра ${ }^{238} \mathrm{U}$ приводит к барьерам $B_{1}=185.4$ МэВ и $B_{2}=199.2$ МэВ для двух предельных ориентаций ядер. Используя полуэмпирическую модель слияния ядер и аппроксимируя функцию $F(B)$ в (30) асимметричным гауссианом с ширинами $\Delta_{B}^{1}=\left(B_{2}-B_{1}\right) / 2 \approx 7$ МэВ для правой ветви (большие значения $B$ ) и $\Delta_{B}^{2}=5$ МэВ для левой ветви (здесь это фактически подгоночный параметр), мы также получаем вполне удовлетворительное согласие с экспериментом - штрихпунктирная кривая на рис. 6.

Квадрат модуля двумерной волновой функции относительного движения ядер ${ }^{48} \mathrm{Ca}{ }^{238} \mathrm{U}$ показан на рис. 7 в зависимости от расстояния $r$ и угла вращения $\theta$ деформированного ядра урана. На этом же рисунке пунктиром показан ландшафт потенциальной энергии взаимодействия двух ядер, аналогичный тому, что изображен на рис. 2а. Выбранная энергия столкновения $E_{c m}=194$ МэВ выше высоты кулоновского барьера при $\theta=0^{\circ}$ ( 185 МэВ) и ниже барьера при $\theta=\pi / 2$ ( 200 МэВ). В результате, как видно из рисунка, падающая волна почти беспрепятственно преодолевает барьер лишь при малых $\theta$ (в конфигурации «нос к носу») и почти полностью отражается от барьера при $\theta \sim \pi / 2$. На больших расстояниях это приводит к сильной интерференции падающей и отраженной волн при $\theta \sim \pi / 2$ и почти к полному отсутствию интерференции при малых $\theta$.

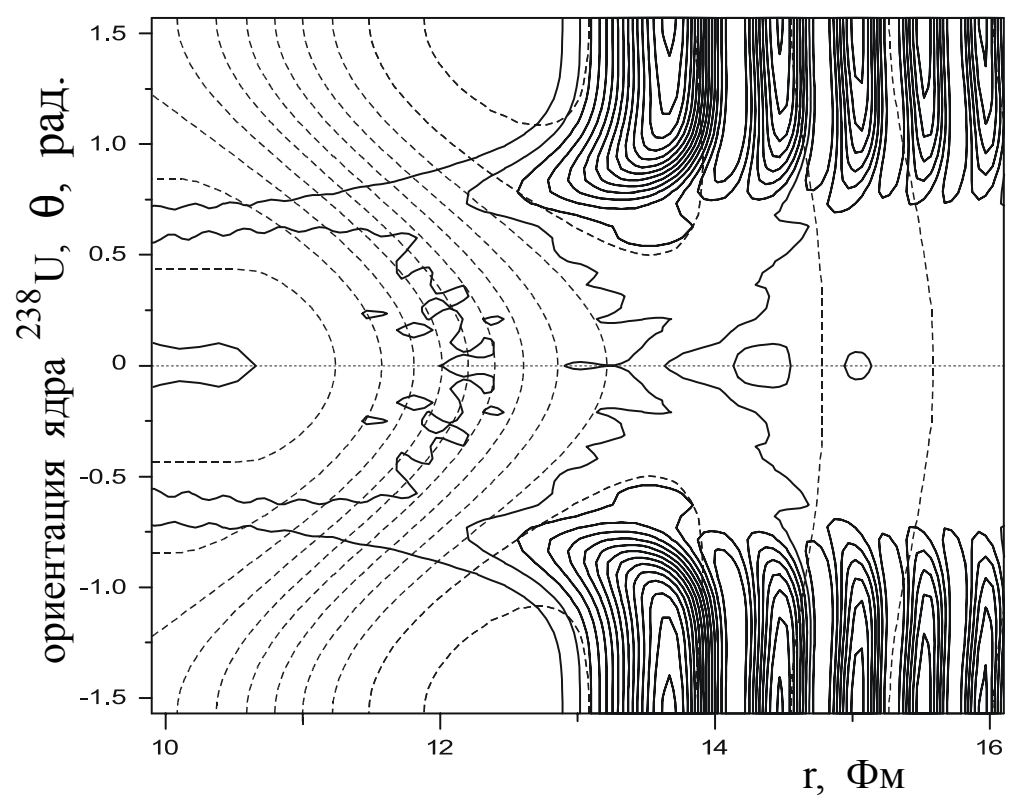

Pис. 7. Топографический ландшафт квадрата модуля двумерной волновой функции (13) для $l=0$, описывающей процесс слияния ядер ${ }^{48} \mathrm{Ca}$ и ${ }^{238} \mathrm{U}$ при энергии $E_{c m}=194 \mathrm{MэB} \mathrm{(сплошные}$ кривые). Ландшафт потенциальной энергии показан пунктиром 


\section{Приложение 1. Локальная кривизна поверхности деформированного ядра и геометрический фактор}

Локальная кривизна плоской кривой, представленной в полярных координатах, определяется выражением ([11], формула 17.1-9)

$$
k=\left[\rho^{2}+2\left(\frac{d \rho}{d \varphi}\right)^{2}-\rho \frac{d^{2} \rho}{d \varphi^{2}}\right]\left[\rho^{2}+\left(\frac{d \rho}{d \varphi}\right)^{2}\right]^{-3 / 2} .
$$

В случае динамических деформаций вдоль межъядерной оси (ориентация «нос к носу») любая кривая, лежащая на поверхности ядра в плоскости, проходящей через ось симметрии, определяется формулой (1), т.е. $\rho(\varphi) \equiv R(\theta)$. Для малых углов $\theta<<1$ можно использовать приближенное выражение для полиномов Лежандра

$$
P_{\lambda}(\cos \theta) \approx 1-\eta(\lambda)(1-\cos \theta), \quad \theta<<1,
$$

где $\eta(\lambda)=3 \cdot 4 \cdot \ldots \cdot(\lambda+1) /(\lambda-1)$ ! и уравнение поверхности имеет вид

$$
R(\theta)=a+b \cos \theta,
$$

где $\quad a=\tilde{R} \cdot\left[1+\sum_{\lambda \geq 2} \beta_{\lambda}(1-\eta(\lambda)) \sqrt{\frac{2 \lambda+1}{4 \pi}}\right], \quad b=\tilde{R} \cdot\left[1+\sum_{\lambda \geq 2} \beta_{\lambda} \eta(\lambda) \sqrt{\frac{2 \lambda+1}{4 \pi}}\right] . \quad$ Подстановка выражения (П.2) в (П.1) дает при $\theta=0$

$$
\begin{gathered}
k^{\perp}=k^{\|}=k=\frac{a+2 b}{(a+b)^{2}}, \\
k=\tilde{R}^{-1}\left(1+\sum_{\lambda \geq 2} \sqrt{\frac{2 \lambda+1}{4 \pi}} \beta_{\lambda}\right)^{-2}\left(1+\sum_{\lambda \geq 2}(1+\eta(\lambda)) \sqrt{\frac{2 \lambda+1}{4 \pi}} \beta_{\lambda}\right),
\end{gathered}
$$

откуда и следует формула (7).

В случае вращающихся в плоскости реакции статически деформированных ядер вычисление локальной кривизны для произвольной ориентации осей симметрии приводит к более громоздким формулам. Простое выражение получается для предельного случая $\theta=\pi / 2$ (ориентация «бок к боку»). Поскольку наиболее характерной для ядер является квадрупольная статическая деформация, то мы рассмотрим здесь лишь случай $\lambda=2$. Используя выражение $1 / 4(3 \cos 2 \theta+1)$ для полинома Лежандра $P_{2}(\cos \theta)$, имеем

$$
R(\theta)=a^{\prime}+b^{\prime} \cos 2 \theta,
$$

где $a^{\prime}=\tilde{R} \cdot\left[1+\frac{1}{4} \beta_{2} \sqrt{\frac{5}{4 \pi}}\right], b^{\prime}=\frac{3}{4} \tilde{R} \cdot \beta_{2} \sqrt{\frac{5}{4 \pi}}$. Подстановка (П.5) в (П.1) при $\theta=\pi / 2$ дает

$$
k^{\|}=\frac{a^{\prime}-5 b^{\prime}}{\left(a^{\prime}-b^{\prime}\right)^{2}}=\frac{R_{A}}{R_{B}^{2}}, \quad k^{\perp}=\frac{1}{R_{B}},
$$


где $\quad R_{A}=\tilde{R} \cdot\left[1-\frac{7}{2} \beta_{2} \sqrt{\frac{5}{4 \pi}}\right], \quad$ а $\quad R_{B}=R(\theta=\pi / 2)=\tilde{R} \cdot\left[1-\frac{1}{2} \beta_{2} \sqrt{\frac{5}{4 \pi}}\right]$. Дальнейшее вычисление локального геометрического фактора $G_{l o c}=P_{s p h} / P$ осуществляется с помощью формулы (6), которая, как уже отмечалось, приводит к сингулярности при соприкосновении двух плоских поверхностей $\left(k_{1}^{\perp}+k_{2}^{\perp}=0\right.$ и/или $\left.k_{1}^{\|}+k_{2}^{\|}=0\right)$.

\section{Приложение 2. Геометрический фактор с учетом конечности действия ядерных сил и размеров деформированных ядер}

Точное значение геометрического фактора, в принципе, может быть получено при использовании фолдинг-процедуры для вычисления ядро-ядерного потенциала. Конечность размеров ядер и радиуса действия нуклон-нуклонного взаимодействия гарантируют в этом случае отсутствие каких-либо сингулярностей, в том числе и для нулевой локальной кривизны в точке касания. В конкретных приложениях, однако (особенно в динамических расчетах), вместо фолдинг-потенциалов удобнее использовать некоторое аналитическое выражение для ядро-ядерного взаимодействия (типа потенциала Вудса-Саксона или потенциала «proximity»), учитывающего, тем не менее, изменение кривизны ядерных поверхностей при больших деформациях. Приближенное значение геометрического фактора может быть получено с помощью выражения

$$
G=\frac{\Delta V_{1}\left(\vec{\beta}_{1}, \theta_{1} ; a_{1}\right)+\Delta V_{2}\left(\vec{\beta}_{2}, \theta_{2} ; a_{2}\right)}{\Delta V_{1}^{0}\left(a_{1}\right)+\Delta V_{2}^{0}\left(a_{2}\right)},
$$

где $\Delta V_{i}\left(\vec{\beta}_{i}, \theta_{i} ; a_{i}\right)$ - объемы наиболее близко расположенных друг к другу малых сегментов ядер (см. рис. 8), а $V_{i}^{0}\left(a_{i}\right)=(\pi / 3) a_{i}^{2}\left(3 R-a_{i}\right)$ - объемы сегментов соответствующих сферических ядер, $a_{1} / a_{2}=\left(R_{2}-a / 2\right) /\left(R_{1}-a / 2\right)$ и $a \approx 1$ Фм. Рассмотрим вначале деформации вдоль межъядерной оси (Рис. 8,a). Объем «носового» сегмента определяется в этом случае выражением

$$
\Delta V_{i}=\frac{2 \pi}{3} \tilde{R}_{i}^{3} \int_{t_{0}^{i}}^{1}\left[1+\sum_{\lambda} \beta_{\lambda}^{i} \sqrt{\frac{2 \lambda+1}{4 \pi}} P_{\lambda}(t)\right]^{3} d t-\frac{\pi}{3}\left(R_{i}^{0}-a_{i}\right)^{3}\left(1 / t_{0}^{i^{2}}-1\right),
$$

где $R_{i}^{0}=R_{i}\left(\vec{\beta}_{i}, \theta=0\right)$, а $t_{0}^{i}$ находится из уравнения $(t \equiv \cos \theta)$

$$
R(t) \cdot t=R_{i}^{0}-a_{i} .
$$

Решение этого уравнения может быть получено в явном виде, если воспользоваться параболической аппроксимацией полиномов Лежандра в области $0<t \leq 1$

$$
P_{\lambda}(t) \approx-p_{\lambda}+\alpha_{\lambda}\left(t-\tau_{\lambda}\right)^{2}
$$

где $p_{\lambda}=\{1 / 2, \sqrt{1 / 5}, 3 / 7\}, \tau_{\lambda}=\{0, \sqrt{1 / 5}, \sqrt{3 / 7}\}$ и $\alpha_{\lambda}=\left(1+p_{\lambda}\right) /\left(1-\tau_{\lambda}\right)^{2}$ для $\lambda=2,3,4$. В этом случае (П.9) является кубическим уравнением и для $t_{0}^{i}$ следует взять положительный корень этого уравнения. Интегрирование в (П.8) также выполняется в явном виде, давая хоть и громоздкое, но легко вычисляемое выражение для объема «носового» сегмента деформированного ядра. Очевидно, 
что геометрический фактор (П.7) не имеет сингулярностей и остается конечной величиной при любых деформациях.

При больших отрицательных деформациях поверхность ядра в точке $\theta=0$ вначале становится плоской, а затем вогнутой (рис. 8,б). Толщину «взаимодействующего слоя» $a$ в этом случае надо отсчитывать не от точки $R_{i}^{0}=R_{i}\left(\vec{\beta}_{i}, \theta=0\right)$, лежащей на оси симметрии, а от точки поверхности, наиболее удаленной от центра ядра вдоль межъядерной оси, т.е. от точки $R_{i}^{C} \equiv R_{i}\left(t_{C}^{i}\right) \cdot t_{C}^{i}$ на рис. 8,б, которая находится из условия $d[R(t) t] /\left.d t\right|_{t_{C}}=0$. Величина $t_{0}^{i} \equiv \cos \theta_{0}^{i}$ при этом по-прежнему определяется из уравнения (П.9), в котором $R_{i}^{0}$ заменяется на $R_{i}^{C}$. На рис. 9 показано сравнение геометрического фактора, рассчитанного по формуле (П.7), с локальным приближением $G_{l o c}=P_{s p h} / P$. Видно, что в области положительных и небольших отрицательных деформаций приближение «конечных сегментов» слабо зависит от величины $a$ и практически не отличается от локального приближения.
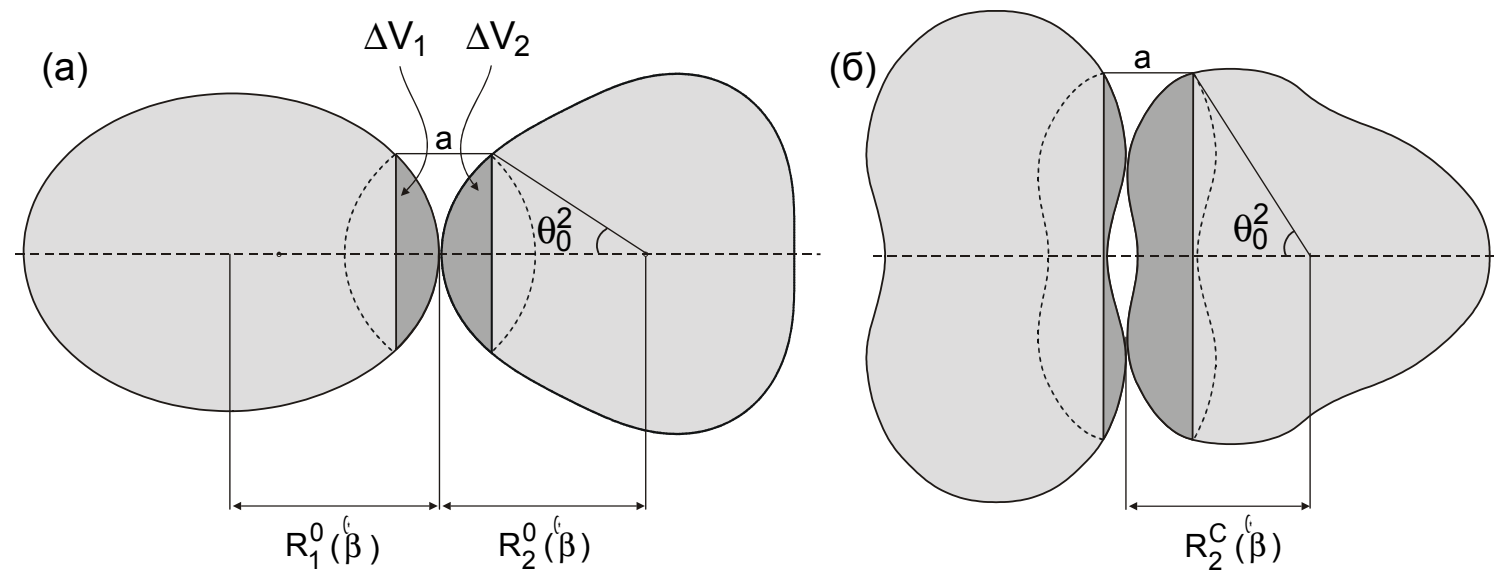

Рис. 8. Схематическое изображение двух деформированных ядер в геометрии «нос к носу» (пунктирная линия - ось вращения). Более темные сегменты показывают области, вносящие основной вклад в ядро-ядерное взаимодействие. (а) - положительные деформации, (б) - отрицательные деформации

Для деформированных вращающихся ядер объемы «взаимодействующих сегментов» $\Delta V_{i}\left(\vec{\beta}_{i}, \theta_{i} ; a_{i}\right)$ зависят от ориентации осей симметрии ядер. Как уже отмечалось, наиболее характерной для ядер является квадрупольная статическая деформация, т.е. $\beta_{3,4}^{\text {g.s. }} \ll \beta_{2}^{\text {g.s. }}$. Кроме этого, при $a \approx 1$ Фм для более высоких мультиполей отклонение объемов $\Delta V_{i}\left(\vec{\beta}_{i}, \theta_{i} ; a_{i}\right)$ от сферических намного меньше, чем для $\lambda=2$. Поэтому при вычислении угловой зависимости геометрического фактора можно ограничиться учетом лишь квадрупольных статических деформаций взаимодействующих ядер. Для вычисления объема бокового сегмента ядра (ориентация ядер «бок к боку», $\theta_{i}=\pi / 2$ ) можно опять использовать 
формулу (П.9) для нахождения угла $\theta_{0}^{i}\left(t_{0}^{i} \equiv \cos \theta_{0}^{i}\right)$ соответствующего конуса. В этой формуле, однако, надо заменить $R_{i}^{0}=R_{i}\left(\vec{\beta}_{i}, \theta=0\right)$ на $R_{i}^{B}=R_{i}\left(\vec{\beta}_{i}, \theta=\pi / 2\right)$. Основание сегмента в этом случае представляет собой эллипс с полуширинами $A=\sqrt{2 R_{i}^{B} a_{i}-a_{i}^{2}}$ и $B=\left(R_{i}^{B}-a_{i}\right) / \operatorname{tg} \theta_{0}^{i}$, а его объем приблизительно равен

$$
\Delta V_{i}=\frac{\pi}{2} a_{i}^{2} \sqrt{2 R_{i}^{B}-a_{i}}\left(R_{i}^{B}-a_{i}\right) / \operatorname{tg} \theta_{0}^{i} .
$$

Зная объемы поверхностных сегментов при двух предельных ориентациях - $\Delta V_{i}^{N}$ (вычисленный по формуле (П.8) для $\left.\theta_{i}=0\right)$ и $\Delta V_{i}^{S}$ (вычисленный по формуле (П.11) для $\left.\theta_{i}=\pi / 2\right)$, можно аппроксимировать объем сегмента произвольно повернутого ядра простым выражением $\Delta V_{i}\left(\theta_{i}\right)=0.5 \cdot\left(\Delta V_{i}^{S}+\Delta V_{i}^{N}\right)-0.5 \cdot\left(\Delta V_{i}^{S}-\Delta V_{i}^{N}\right) \cos 2 \theta_{i} \quad$ и $\quad$ далее $\quad$ воспользоваться формулой (П.7) для вычисления геометрического фактора в потенциале взаимодействия двух деформированных ядер, произвольно повернутых в плоскости реакции.

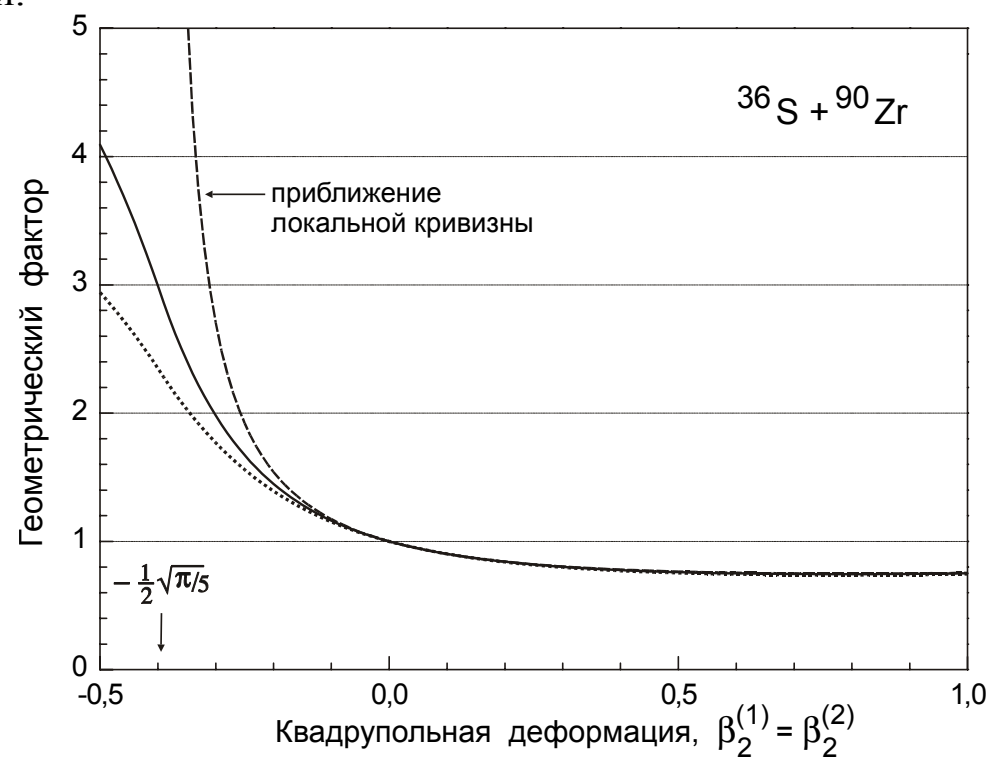

Рис. 9. Геометрический фактор, рассчитанный для случая одинаковых динамических квадрупольных деформаций взаимодействующих ядер ${ }^{36} \mathrm{~S}$ и ${ }^{90} \mathrm{Zr}$. Пунктирная кривая соответствует локальному приближению $G_{l o c}=P_{s p h} / P$. Сплошная и точечная кривые рассчитаны по формуле (П.7) с $a=1$ ФМ и $a=2$ ФМ соответственно. Стрелкой показана «критическая» деформация, при которой локальная кривизна поверхности с квадрупольной деформацией обращается в ноль (соприкосновение двух плоских поверхностей)

В заключение отметим, что разработанные нами и используемые в данной работе алгоритмы расчета сечений околобарьерного слияния тяжелых ядер (метод связанных каналов и полуэмпирическая модель) вместе с программой расчета многомерных потенциальных поверхностей размещены на Web-сервере [26] со свободным доступом.

Работа была поддержана грантом РФФИ 01-07-90191. 


\section{Литература}

[1] M. Beckerman, Rep. Prog. Phys., 51 (1988) 1047 (см. также ссылки).

[2] Heavy Ion Fusion, Eds. A.M. Stefanini et al., World Scientific, Singapore, 1994.

[3] Papers from Fusion 97, J. Phys. G: Nucl. Phys., 23 (1997) 1157-1501.

[4] M. Dasgupta, D.J. Hinde, N. Rowley, A.M. Stefanini, Annu. Rev. Nucl. Part. Sci., 48 (1998) 401.

[5] C.H. Dasso, S. Landowne, Comp. Phys. Commun., 46 (1987) 187; J. Fernandez-Niello, C.H. Dasso, S. Landowne, Comp. Phys. Commun., 54 (1989) 409.

[6] K. Hagino, N. Rowley, A.T. Kruppa, Comp. Phys. Commun., 123 (1999) 143.

[7] В.И. Загребаев, Н.С. Николаева, В.В. Самарин, Изв. АН (Сер. Физ.), 61 (1997) 2157.

[8] N. Takigawa, T. Rumin, N. Ihara, Phys. Rev., C 61 (2000) 044607.

[9] J. Blocki, J. Randrup, W.J. Swiatecki, C.F. Tsang, Ann. Phys. (N.Y.), 105 (1977) 427.

[10] R.A. Broglia, C.H. Dasso, and A. Winter, Proceedings of Int. School of Physics "Enrico Fermi”, Varenna Course 1979, Eds. R.A. Broglia, R.A. Ricci, H.A. Dasso, North-Holland, Amsterdam, 1981, c.327.

[11] Г. Корн, Т. Корн, Справочник по математике для научных работников и инженеров, «Наука», Москва, 1970.

[12] I.I. Gontchar, M. Dasgupta, D.J. Hinde, R.D. Butt, A. Mukherjee. Phys. Rev., C 65 (2002) 034610 .

[13] О. Бор, Б. Моттельсон. Структура атомного ядра, «Мир», Москва, 1977, Т.2.

[14] M.A. Nagarajan, N. Rowley, R.J. Lindsay, J. Phys. G: Nucl. Phys., 12 (1986) 529.

[15] Дж. Уилкинсон, Г. Райнш, Справочник алгоритмов на языке АЛГОЛ: Линейная алгебра, М.: Машиностроение, 1976.

[16] J. Tôke, R. Bock, G.X. Dai, S. Gralla, A. Gobbi, K.D. Hildenbrand, J. Kuzminski, W.F.J.Müller, A. Olmi, H. Stelzer, B.B. Bock, S. Bjornholm, Nucl. Phys., A440 (1985) 327.

[17] V.I. Zagrebaev, Phys. Rev., C 64 (2001) 034606.

[18] Современные численные методы решения обыкновенных дифференциальных уравнений, под редакцией Дж. Холла и Дж. Ватта, «Мир», Москва, 1979.

[19] J.R. Leigh et al., Phys. Rev., C 52 (1995) 3151.

[20] N. Rowley, G.R. Satchler, P.H. Stelson, Phys. Lett., B 254 (1991) 25.

[21] D.L. Hill, J.A. Wheeler, Phys. Rev., 89 (1953) 1102.

[22] F. Scarlassara, S. Beghini, G. Montagnoli, G.F. Segato, L. Corradi, A.M. Stefanini, A.M. Vinodkumar, in Proceedings on Fusion Dynamics at the Extremes, Dubna, 2000, edited by Yu.Ts. Oganessian and V.I. Zagrebaev, World Scientific, Singapore, 2001, p.274.

[23] M.G. Itkis et al., in Proceedings on Fusion Dynamics at the Extremes, Dubna, 2000, edited by Yu.Ts. Oganessian and V.I. Zagrebaev, World Scientific, Singapore, 2001, p.93.

[24] R. Bass, Nuclear Reactions with Heavy Ions, Springer-Verlag, Berlin, 1980, p.326.

[25] P. Möller, J.R. Nix, W.D.Myers, W.J.Swiatecki, At. Data Nucl. Data Tables, 59 (1995) 185.

[26] http://nrv.jinr.ru/nrv/ 